\title{
How different are different diff algorithms in Git?
}

$$
\text { Use - - histogram for code changes }
$$

\section{Yusuf Sulistyo Nugroho $^{1}$ (i) Hideaki Hata $^{1} \cdot$ Kenichi Matsumoto $^{1}$}

Published online: 11 September 2019

(C) The Author(s) 2019

\begin{abstract}
Automatic identification of the differences between two versions of a file is a common and basic task in several applications of mining code repositories. Git, a version control system, has a diff utility and users can select algorithms of diff from the default algorithm Myers to the advanced Histogram algorithm. From our systematic mapping, we identified three popular applications of diff in recent studies. On the impact on code churn metrics in 14 Java projects, we obtained different values in $1.7 \%$ to $8.2 \%$ commits based on the different diff algorithms. Regarding bug-introducing change identification, we found $6.0 \%$ and $13.3 \%$ in the identified bug-fix commits had different results of bug-introducing changes from 10 Java projects. For patch application, we found that the Histogram is more suitable than Myers for providing the changes of code, from our manual analysis. Thus, we strongly recommend using the Histogram algorithm when mining Git repositories to consider differences in source code.
\end{abstract}

Keywords Code changes $\cdot$ Diff $\cdot$ Histogram algorithm $\cdot$ Mining repositories

\section{Introduction}

The diff utility calculates and displays the differences between two files, and is typically used to investigate the changes between two versions of the same file. Since understanding and measuring changes in software artifact is essential in empirical software engineering research, diff is commonly used in various topics, such as defect prediction where code churn (Nagappan and Ball 2005; Shin et al. 2011) and process metrics (Hata et al. 2012;

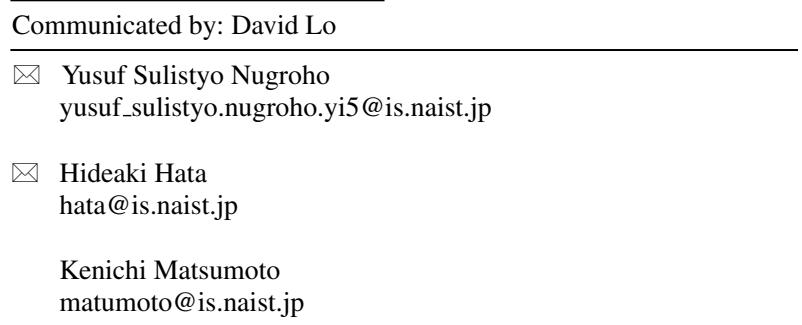

1 Nara Institute of Science and Technology, Ikoma, Japan 
Madeyski and Jureczko 2015; Kamei and Shihab 2016) are used, code authorship (Rahman and Devanbu 2011; Meng et al. 2013), clone genealogy (Kim et al. 2005; Duala-Ekoko and Robillard 2007), and empirical studies of changes (Barr et al. 2014; Ray et al. 2015).

Along with the growth of GitHub, recent studies analyze software changes from Git repositories by using the git command. Git, a version control system, offers diff utility for users to select the algorithms of diff. Git offers four diff algorithms, namely, Myers, Minimal, Patience, and Histogram. Without an identifying algorithm, Myers is used as the default algorithm.

In textual differencing, all diff algorithms are computationally correct in generating the diff outputs. However, the diff outputs are sometimes different due to different diff algorithms. Different diff algorithms might identify different change hunks, that is, a list of program statements deleted or added contiguously, separated by at least one line of unchanged context (Ray et al. 2015). We expect that a set of changing operations done by developers can be represented by change hunks. However, there can be inappropriate identifications of change hunks. Although Histogram that was introduced in git $1.7 .7^{1}$ in 2011 might give better performance to git diff, it is not popular among software engineer communities. Thus, we focus on the Myers and Histogram algorithms to empirically investigate the impact on software engineering research. The motivation of this study is try to clarify the impact of adopting different diff algorithms on empirical studies and investigate which diff algorithm can provide better diff results that can be expected to recover the changing operations. Furthermore, our study provides a comprehensive procedure of Myers and Histogram in generating the diffs and shows the differences between their outputs. To the best of our knowledge, empirical comparisons of different diff algorithms in git diff command have never been undertaken. In this paper, we carry out two sequential analyses: systematic mapping and empirical comparisons.

For the systematic mapping, we collect papers from three high ranking journals and eight top international conference proceedings published from 2013 to 2017 . We then map 52 identified papers in the following four aspects: frequency of diff algorithms, analyzed software artifact, purpose of mining Git repositories, and data origins. The results of the systematic mapping revealed that the advanced diff algorithms had not been considered in the previous studies. In terms of the focus of the git command, 51 out of 52 papers centralized on mining the code changes. We also found that the purposes of using the git command were to get patches $(46.2 \%)$, followed by metrics collection $(25 \%)$, and bug-introducing change identification (SZZ algorithm) (23.1\%). Regarding the dataset, most papers investigated OSS projects $(98 \%)$, even though the remaining work analyzed industrial data.

In our empirical analyses, we conduct three comparisons based on the most popular usages of git diff found in our mapping study: collecting metrics, identifying bug introduction, and getting patches. We investigate the disagreement between two diff algorithms: Myers and Histogram, and take a manual measurement of their quality in generating the diff lists. Based on previous related studies, we investigate the code changes from the files in 14 OSS projects that employ Continuous Integration for metrics collection and 10 Apache projects for the bug introduction identification to quantify the differences of the diff outputs that resulted from both diff algorithms. We analyze the quality of patches derived from Myers and Histogram by manually comparing their two diff from

\footnotetext{
${ }^{1}$ https://github.com/git/git/blob/77bd3ea9f54f1584147b594abc04c26ca516d987/Documentation/RelNotes/ 1.7.7.txt\#L68-L70
} 
377 changes, a statistically representative sample of the 21,590 changes identified in the above two comparisons. Our findings show that using various diff algorithms in the git diff command produced unequal diff lists.

This influences the different number of files that have dissimilar added and deleted lines of code in each CI-Java project. The differences of these added and deleted lines that are distinguished by their different number and position range from $0.8 \%$ to $6.2 \%$ and from $1.4 \%$ to $7.6 \%$, respectively. The divergent diff outputs also affected the different number of identified files in bug introduction identification. The percentage of files that have different deleted lines of code range from $2.4 \%$ to $6.6 \%$. Regarding the result of the patches analysis, we found that, in-code changes, Histogram is better in $62.6 \%$ files, while Myers is better in $16.9 \%$ files. However, both diff algorithms evenly have a good quality in generating the list of non-code changes.

In sum, the contributions of this work are:

- A systematic survey of studies that use diff;

- An analysis of metrics collected from diff outputs produced by Myers and Histogram;

- An analysis of Myers and Histogram outputs in identifying potential bug-introducing changes;

- A manual comparison between Myers and Histogram to investigate their output quality.

The remaining parts of this paper are structured as follows. Section 2 presents the application of various category of diff algorithms in the literature. Section 3 presents a brief explanation of diff algorithms used in the git command. We explain the differences between two diff algorithms in generating the list of changes. Section 4 describes how we conduct a systematic mapping study and present the result of the survey. The overview of the three comparisons and the research questions are presented in Section 5. Sections 6, 7 and 8 report our procedures and discuss their results in performing three comparison studies; namely, collecting metrics, identifying bug introduction, and getting patches respectively. In Section 9, we discuss the implication of different diff algorithms and provide the example, and discuss their threats to validity, and finally we conclude in Section 10.

We have provided the data sets used in this paper publicly on the Web. ${ }^{2}$

\section{Source Code Differencing}

Existing differencing techniques use similarities in names and structure to match code elements at a particular granularity, such as text-based and abstract-syntax-tree-based (AST).

Tree-based differencing techniques are widely used nowadays (e.g., diff in Unix), since they are expected to have better understandability than the text-based. Such AST differencing tools were used in several studies. For example, Change Distilling (CD) that extracts the code changes by finding both a match between the nodes of the compared two abstract syntax trees and a minimum edit script that can transform one tree into the other given the computed matching (Fluri et al. 2007). In this study, the text-based differencing is used to extract the changes at the beginning of the process as the input before further processed using the proposed AST algorithm. In comparison with textual diff, the Change Distiller is able to assign the type of the changes such as declaration or body part of a method,

$\overline{{ }^{2} \text { https://github.com/yusufsn/DifferentDiffAlgorithms }}$ 
rather than just to a line number. Diff/TS (Hashimoto and Mori 2008) and MTDIFF (Dotzler and Philippsen 2016) use moving code to compute the changes. Diff/TS is used to analyze fine-grained structural change between versions of programs but only capable of processing Python, Java, $C$, and $C++$ projects, while MTDIFF improves the accuracy of the previous tree-based approaches in detecting moved code. Falleri et al. (2014) introduced an algorithm to compute edit scripts at the abstract syntax tree granularity including move actions. In this study, the authors conducted a performance study to measure the running time and memory consumption between their proposed algorithm and the other tools, such as GumTree and RTED algorithm. The classical text diff was used to present the reference values when comparing the running time between the involved algorithms. Tree-based differencing approach was also used by Higo et al. (2017) to consider copy-and-paste as a type of editing action forming tree-based edit script, and Huang et al. (2018) to propose CLDIFF for generating concise linked code differences whose granularity is in between the existing code differencing and code change summarization methods.

Despite many advantages in tree-based differencing techniques, text-based diff is widely used for several applications in software engineering research because of its simplicity and lightweight runtime. Therefore, in this paper we only focus on studying the impact of changing diff algorithms, instead of comparing wider categories of differencing techniques.

\section{Diff Algorithms in Git}

Diff is an automatic comparison program used to find the disagreements between the older and the newer version of the same file in a storage (including insertions, deletions, document renaming, document movements etc.). The diff utility extracts code changes line by line in one file compared to the other file and reports them in a list. The operation of the diff program has been fundamentally solved by using the longest common subsequence (LCS) problem initiated by Hunt and MacIlroy (1976). Since its first run on the Unix operating system in 1970, the diff command has been widely used in many studies.

The git diff command has numerous options in the application of code changes extraction, ${ }^{3}$ including extracting changes related to the index and commit, paths on a filesystem, the original contents of objects, or even quantifying the number of changes for each object relatively from the sources. Researchers and practitioners are able to use the variation of these available options depending on their needs in extracting the data, not to mention, the diff algorithms. The essence of diff algorithms is in contrasting the two sequences and to receive insight of the transformation from the first into the second by a series of operations using the ordered deletion and insertion. The subsequence can be flagged as a change if a delete and an insert concur on the same scope. The diff algorithm can be selected with this option - -diff-algorithm=<algorithm>.

In Git, there are four diff algorithms, namely Myers, Minimal, Patience, and Histogram, which are utilized to obtain the differences of the two same files located in two different commits. The Minimal and the Histogram algorithms are the improved versions of the Myers and the Patience respectively. Each algorithm has its own procedures for finding the items presented in the original document, but absent in the second one and vice versa; as a consequence, different outputs may be produced. Due to the similarity of the basic idea of Minimal and Histogram algorithms with their precursors, in this paper we only contrasted the two diff algorithms: Myers and Histogram.

\footnotetext{
${ }^{3}$ https://git-scm.com/docs/git-diff
} 

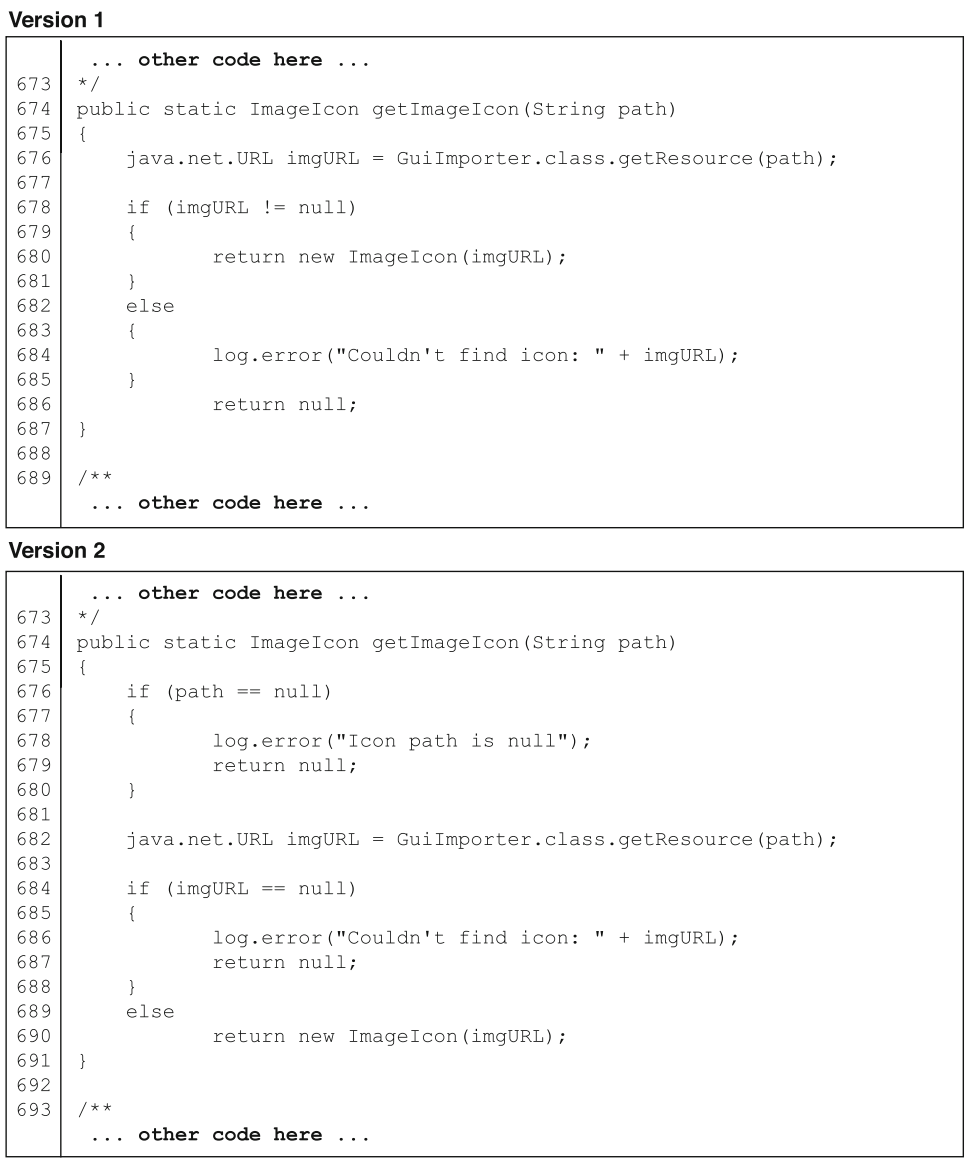

Fig. 1 A set of changes from an older file into a newer file

\subsection{Myers}

Myers algorithm was developed by Myers (1986). In the git diff command, this algorithm is used as the default. The operation of this algorithm traces the two primary identical sequences recursively with the least edited script. Since the Myers only notices the sequences which are actually equal in both, the comparison between the other prior and posterior subsequences is executed repetitively for the entire remaining sequences.

Figure 1 indicates several code changes from the first into the second version of the same file (GuiCommonElements . java) taken from Openmicroscopy project. ${ }^{4}$ As can be seen in the figure, the code between line 673 and 689 in the first version transformed to the newer version between line 673 and 693. Figure 2 shows how Myers algorithm generates the diff output from the code changes in Fig. 1. First, the Myers scans the lines of code sequentially from the first line in both versions of the same file to find a line pair that match

\footnotetext{
${ }^{4}$ https://github.com/openmicroscopy/openmicroscopy/commit/844e0fde447d2d069fb17c480e95acf4d372afc4\# diff-07322c93ef4fb3f0dd245932b74b10e1
} 
up each other. Once the exact same lines between the two versions of the file are found by the algorithm, the lines will be considered as the unmodified lines (e.g. pair of lines 673-675 in both versions in Fig. 2a). The algorithm then do the same scanning to extract the other pairs of matched lines for the remaining lines of code repetitively, as depicted in Fig. $2 b$ and c. In Fig. 2c, we can see all unmodified lines found by the Myers algorithm: pair of line 673-675 in both versions, pair of line 679 in Version 1 and 677 in Version 2, 681 and 680, 683 and 685, 684 and 686, 686 and 687, and 687 and 688). The unpaired lines in Version 1 are subsequently
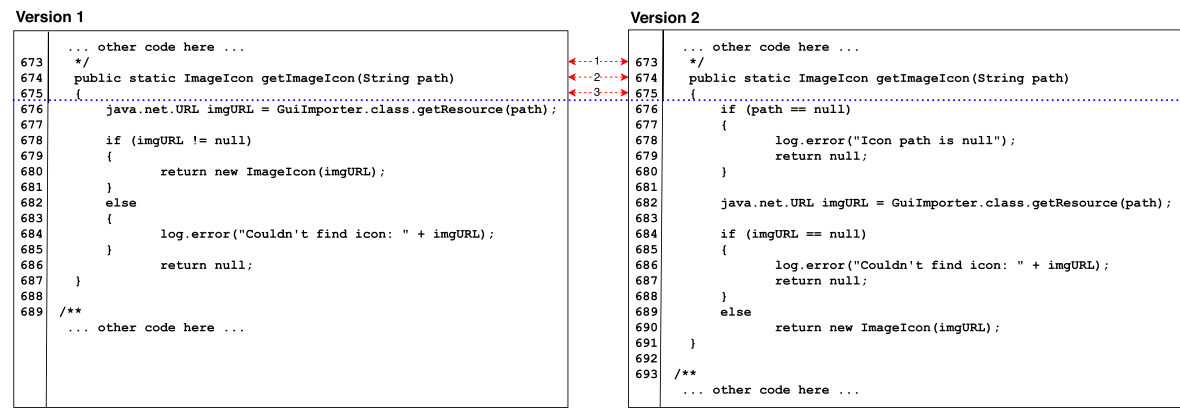

(a) Step 1: pair up the first three matching lines (line 673-675 in both versions)

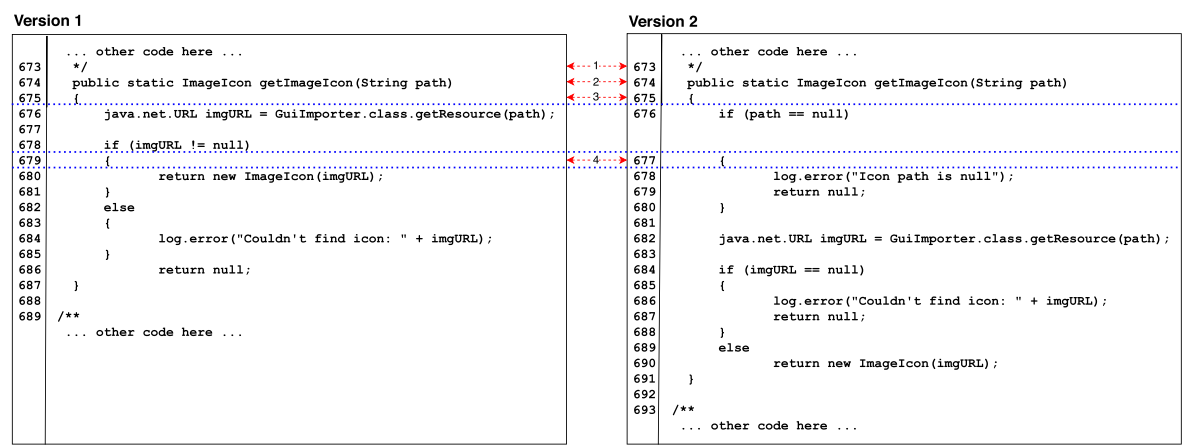

(b) Step 2: pair up the fourth matching lines (line 679 in version 1 and line 677 in version 2)

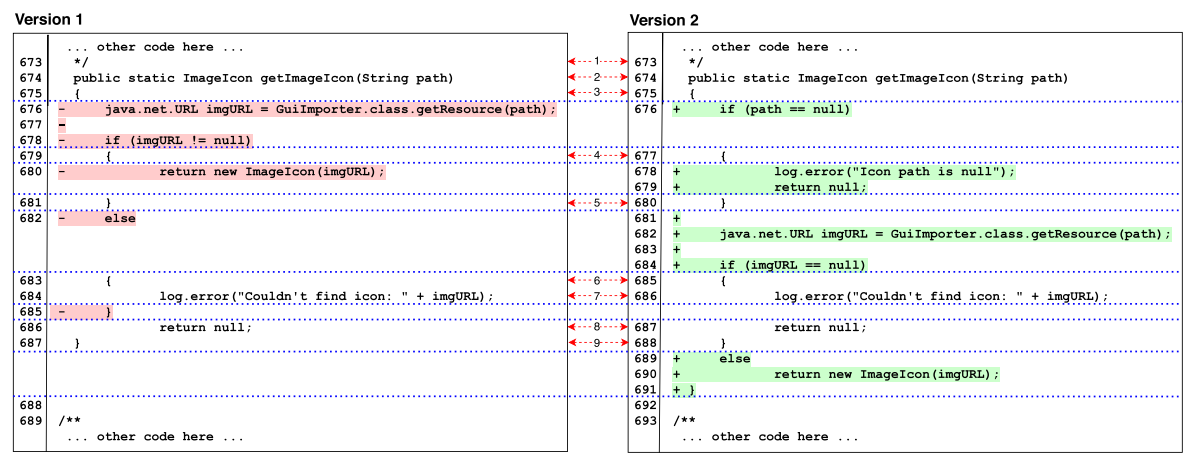

(c) Step n: final step after pairing up all matching lines

Fig. 2 How Myers identifies the diff 
considered as the deleted lines, while the unpaired lines in Version 2 are counted as the added lines. As a result, the Myers algorithm produces the paired and unpaired lines from the first and second version of the same file in sequence, as illustrated in Fig. 4a.

The Minimal algorithm is the extended version of Myers. The operation of this algorithm in finding the changes resulted from a comparison of two objects resembling the Myers, but an extra attempt was made to keep the patch size as minimal as possible. ${ }^{5}$ As a result, the diff lists created using this algorithm are often identical with the Myers. If we apply the Minimal algorithm to the code in Fig. 1, the diff output is shown in Fig. 4a as well.

A major limitation of the Myers algorithm is it frequently catches the blank lines or parentheses and conforms the lines to match instead of catching the line that is "unique" (i.e. lines that occur exactly once or the least occurrence in both versions), such as code of function declaration, or a line of assignment. Consequently, the Myers sometimes produces unclear diff lists that do not describe the actual code changes. The position between changed code and code that replace them is often written distantly in inappropriate lines, or located separately in a line that does not represent the modification. Additionally, there is occasionally a conflict of identification of the changed code; for example, the code in lines 4 and 15 in Fig. 4a. In fact, these lines of code were derived from the same unique line that was unmodified. Using the Myers algorithm, this unique line is detected as a changed code even though it does not show the alteration. This makes it possible to cause misidentification of a code change.

\subsection{Histogram}

The Histogram algorithm is the enhanced version of Patience, which was built by Bram Cohen who is renowned as the BitTorrent developer. ${ }^{6}$ It supports low-occurrence common elements which are applied to improve efficiency. The Histogram was initially built in jgit ${ }^{7}$ and was introduced in git 1.7.7.

The Patience marks the important lines within the text by focusing on the lines that have the smallest number of occurrences, but are essential. This diff automated procedure is an LCS-based problem as well, but it uses a different technique. The Patience only notices the longest common subsequence of the marked lines attained from the lines which emerge uniquely in a specific range and the lines that are also written precisely similar in both files. This implies that the lines having a single bracket or a new line are usually disregarded; otherwise, the Patience retains the distinctive line such as a function definition.

The Histogram strategy works similarly to the Patience by developing a histogram of the appearances for every line in the first version of a file. Every element in the second version is subsequently shown to match with the first sequence in an orderly way to find the existences of the elements and to count the occurrences. If the elements exist and their presences are less than in the first sequence, they are expected to be a potential LCS. Once the screening is finished for the second sequence, the lowest occurrence of LCS is marked as the separator. Two sections resulting from the partition (i.e. section 1 represents the area before the LCS, while section 2 represents the region after the LCS), are then executed repetitively using the same process as the beginning of the algorithm. This means that the Histogram performs similarly to the Patience if a unique common element exists in both files; otherwise, it selects the element that has the least occurrences. In comparison with the

\footnotetext{
${ }^{5}$ http://fabiensanglard.net/git_code_review/diff.php

${ }^{6} \mathrm{https}: / /$ alfedenzo.livejournal.com/170301.html

${ }^{7}$ http://eclipse.org/jgit/
} 


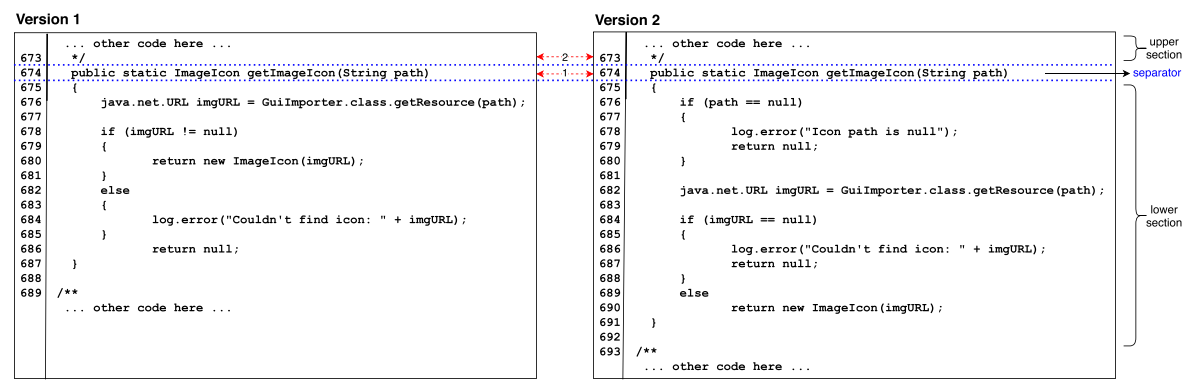

(a) Step 1: pair up the first and second matching unique lines (line 674 in both versions and line 673 at the upper section of the partition)

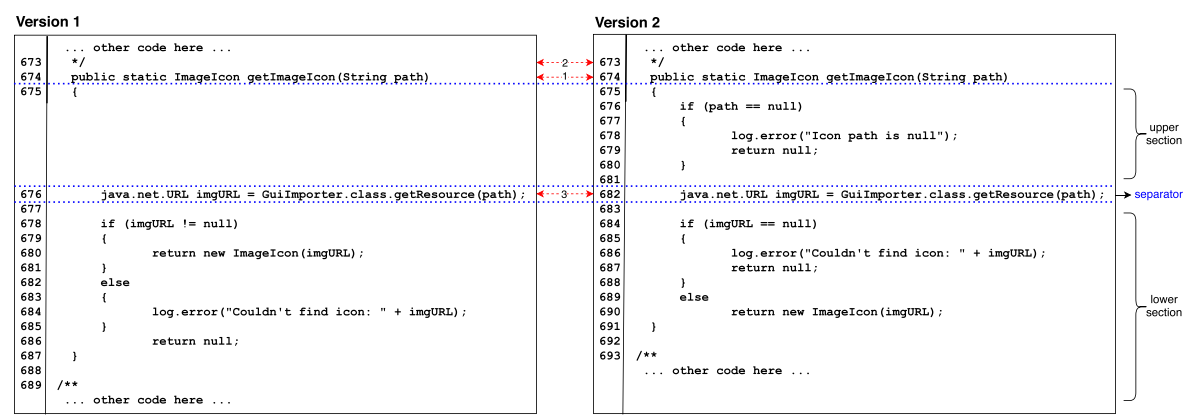

(b) Step 2: pair up the third matching unique line at the lower section of the partition (line 676 in version 1 and line 682 in version 2)

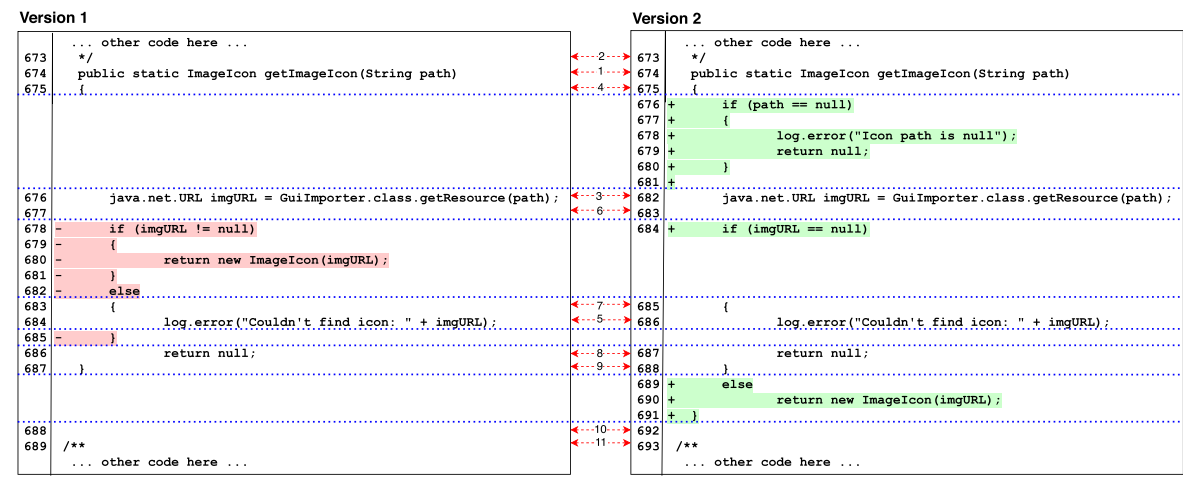

(c) Step n: final step after pairing up all matching unique lines

Fig. 3 How Histogram identifies the diff

other two diff algorithms, (i.e. the Myers and the Patience), the Histogram nevertheless, has been declared much quicker. ${ }^{8}$

To easily understanding the Histogram generates the diff output from Fig. 1, we describe the procedure in Fig. 3. First, the Histogram scans all elements in the first version

$\overline{8_{\text {https: }} / / \text { marc.info/?l=git\&m }=133103975225142 \& w=2}$ 
of the file to count the appearances of each line. Every line in the second version is extracted to match with the element in the first version sequentially to find the exact same line and count the occurrences. If the algorithm found the lines in both versions are match and their presences are unique (i.e. occurs exactly once or have the lowest occurrences in both), they are considered as the potential LCS which is then marked as the separator. As shown in Fig. 3a, line 674 in both versions are marked as the first separator. Two sub-sections are created after this slicing, that is, the area before and after the separator. Within those subsections, the algorithm find more unique pairings; lines that are not unique when scanning the entire document can be unique when the algorithm consider a sub-section. The same process is then applied to both sub-sections. The Histogram compares line 673 in the upper section in both versions, and lines 675-689 in Version 1 with lines 675-693 in Version 2 in the lower sections. Due to the least appearances of line 673 only in the upper section in both versions, thus, this line is expected to be the second separator. In the lower section, the scanning process is re-executed from the beginning. As illustrated in Fig. 3b, the process yields a new separator (i.e. line 676 in Version 1 and line 682 in Version 2) and two new sub-sections (i.e. line 675 in Version 1 and line 675-681 in Version 2 as the upper section, and line 677-689 in Version 1 and line 683-693 in Version 2 as the lower section). The same process is subsequently executed repetitively for the two new sub-sections resulting from the partition. Figure $3 \mathrm{c}$ shows the final step after comparing all elements in both versions. All potential LCS that are marked as the separator are expected to be the unmodified lines, while the other lines are considered as the deleted lines in Version 1 and the added lines in Version 2. As a result, the diff output is generated as described in Fig. 4b.

In contrast with the Myers, the Histogram algorithm provides diff results that are easier for software archives miners to understand, as the Histogram more clearly separates the changed code lines. This algorithm splits the changed lines of code by trying to match up unique lines between two versions of the same file. Thus, it will reduce the occurrences of conflict (i.e. a line of an unchanged code identified as a changed code, so that in the diff list, this code is written in duplicate as both a deleted and inserted code). For example, if we extract the differences between the two versions of the same file in Fig. 1 using the Histogram in the git diff command, we obtain the output as depicted in Fig. 4b. A unique line of code in line 10 of Fig. 4b is not detected as a changed code due to its role as the benchmark to match the line, where this line is identified as a changed code in case of Myers. This influences the sequences of the other changed code. An additional block of if condition is written between lines 4 and 9 where it should be placed. This block of code is clearly understood as the new code inserted before the statement of the assignment code (code in line 10 which is used as one of some unique lines to match). It is also obvious that the code between lines 12 and 16 were replaced by one line of code in line 17, while the closing curly brace in line 20 was omitted from the files, and three new lines of code (line 23, 24 and 25) were added at the end of the code in Fig. 4b.

\section{Systematic Mapping: How Previous Studies Used Git Diff?}

To understand the ways in which the previous studies use diff, we conducted a systematic mapping of papers that used the git diff command for their studies. As described by Petersen et al. (2008), a systematic mapping study can provide and visualize a statistical insight of a study domain by classifying and quantifying the number of publications related to the research interest within the same study domain. The main activity of the method was 


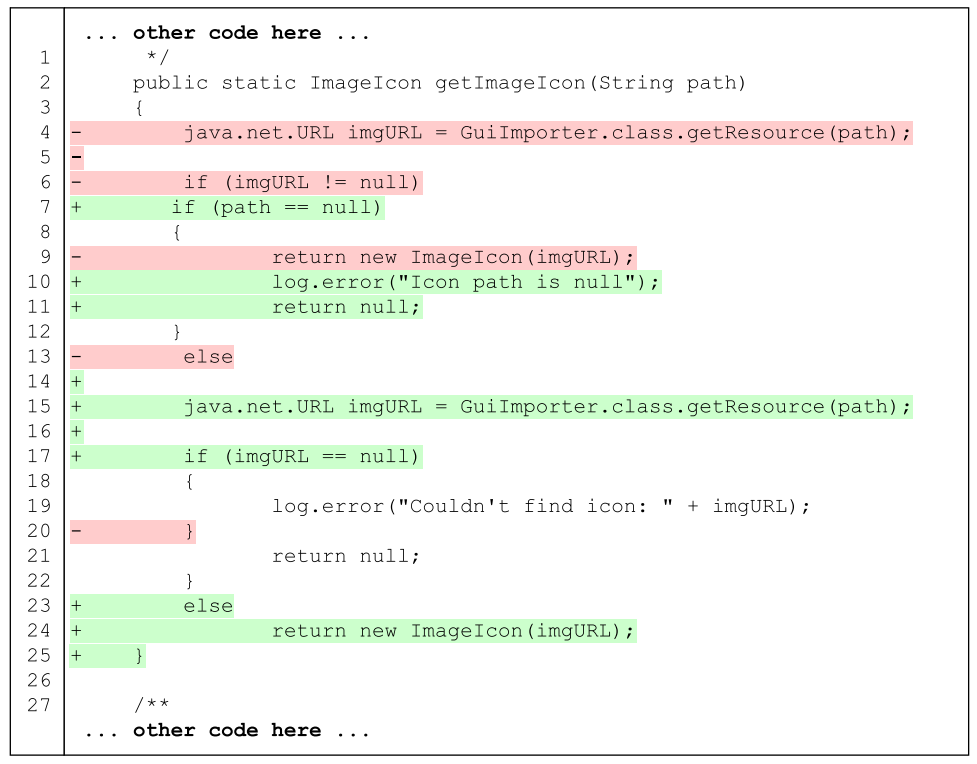

(a) Myers' diff

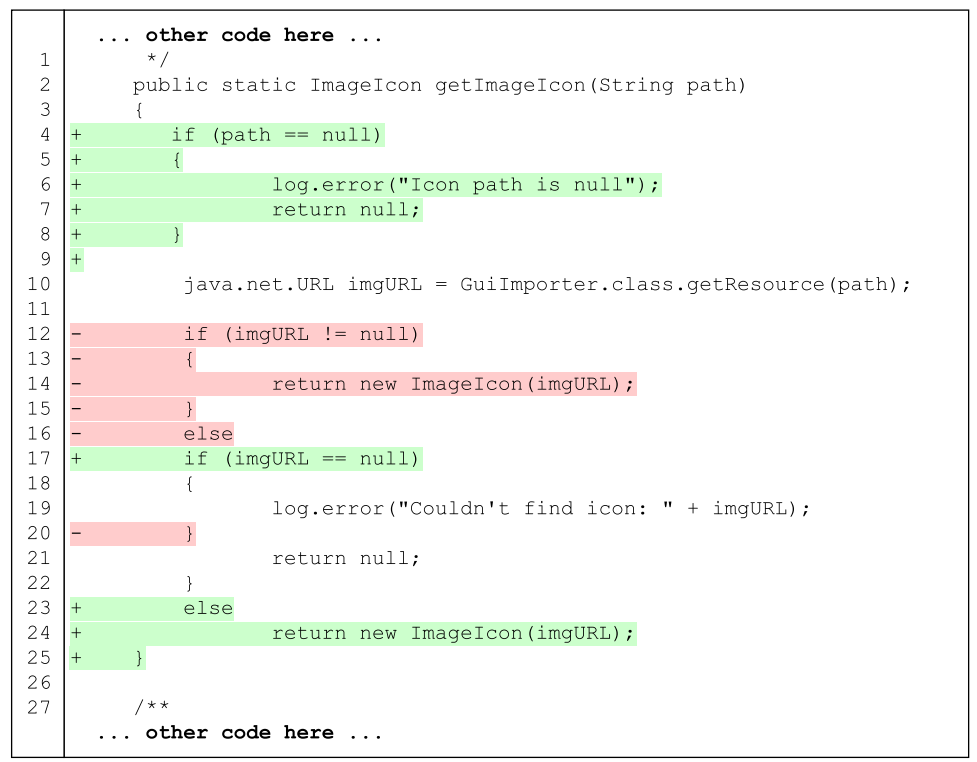

(b) Histogram's diff

Fig. 4 Diff outputs produced by Myers and Histogram

searching the relevant literature from a wide range of publications including journal articles, books, documented archives and scripts.

We performed a systematic mapping as we intend to: (i) draw an overview of the research area through quantification in a structured way (Kuhrmann et al. 2017), (ii) confirm the 


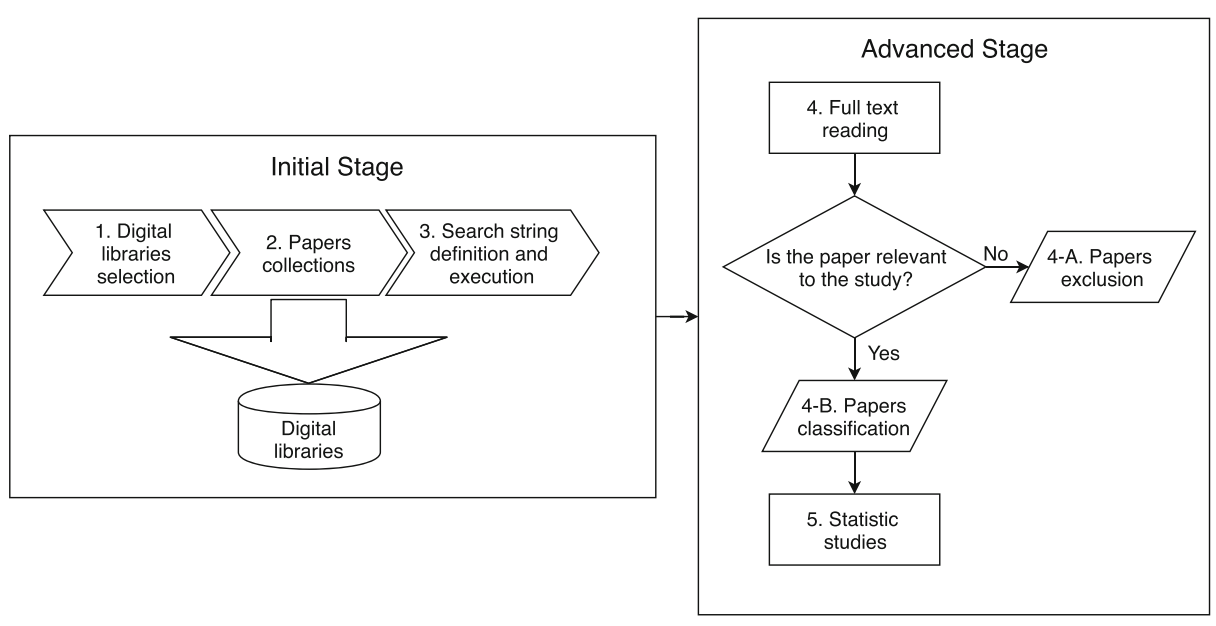

Fig. 5 Design of the survey procedure

knowledge in the currently published studies (Petersen et al. 2015). A systematic mapping is reliable because the findings are repeatable and consistent across the time (Wohlin et al. 2013), and they are beneficial for better reporting of some empirical findings of the primary studies (Budgen et al. 2008).

To understand how recent studies used git diff, we prepared the following research questions for this systematic mapping.

- Which diff algorithm is used?

- What kind of software artifact is analyzed, code or other documents?

- What are purposes of using diff?

- Where does the data source come from, OSS or industry?

\subsection{Procedure}

Figure 5 illustrates an overview of our systematic mapping procedure, which is divided into an initial stage and an advanced stage. The first stage has three steps including a digital libraries selection, papers collection, search string definition and initial search execution. The second stage begins with repetitive manual exclusion by narrowing the search terms and the reading of full papers, followed by paper classification, and statistical analyses.

Step 1: Digital Libraries Selection. The selection of appropriate literature is essential to guarantee high-quality papers and to grasp the state-of-the-art issues in the software engineering field (Kavitha 2009). We specifically targeted papers which were published in high ranking journals and conference proceedings of the software engineering area. To maximize the probability of finding highly relevant good quality articles, we used three specific digital resources: ACM Digital Library, ${ }^{9}$ IEEE Xplore, ${ }^{10}$ and SpringerLink. ${ }^{11}$ Table 1 shows the list of the publication sources used in our survey including their impact

\footnotetext{
${ }^{9}$ https://dl.acm.org/

${ }^{10} \mathrm{https} / / /$ ieeexplore.ieee.org/

${ }^{11} \mathrm{https} / / /$ link.springer.com/
} 
Table 1 List of surveyed SE journals and conferences

\begin{tabular}{|c|c|c|}
\hline Category & Name of Journal or Conference & IF or Rank \\
\hline \multirow[t]{3}{*}{ Journal } & IEEE Transactions on Software Engineering (TSE) & $\mathrm{IF}=3.331$ \\
\hline & Empirical Software Engineering (EMSE) & $\mathrm{IF}=2.933$ \\
\hline & $\begin{array}{l}\text { ACM Transactions on Software Engineering and Methodology } \\
\text { (TOSEM) }\end{array}$ & $\mathrm{IF}=1.946$ \\
\hline \multirow[t]{8}{*}{ Conference } & $\begin{array}{l}\text { ACM Conference on Object Oriented Programming Systems } \\
\text { Languages and Applications (OOPSLA) }\end{array}$ & Rank $=A^{*}$ \\
\hline & $\begin{array}{l}\text { ACM-SIGPLAN Conference on Programming Language } \\
\text { Design and Implementation (PLDI) }\end{array}$ & Rank $=A^{*}$ \\
\hline & International Conference on Software Engineering (ICSE) & Rank $=A^{*}$ \\
\hline & $\begin{array}{l}\text { Joint Meeting of the European Software Engineering Confer- } \\
\text { ence and the ACM SIGSOFT Symposium on the Foundations } \\
\text { of Software Engineering (ESEC/FSE) }\end{array}$ & Rank $=A^{*}$ \\
\hline & Automated Software Engineering (ASE) & Rank $=\mathrm{A}$ \\
\hline & $\begin{array}{l}\text { International Conference on Software Maintenance and Evo- } \\
\text { lution (ICSME) }\end{array}$ & Rank $=\mathrm{A}$ \\
\hline & International Conference on Mining Software Repositories (MSR) & Rank $=\mathrm{A}$ \\
\hline & International Symposium on Software Testing and Analysis (ISSTA) & Rank $=\mathrm{A}$ \\
\hline
\end{tabular}

factors (IF) ${ }^{12}$ and rankings published in 2018 CORE Rankings. ${ }^{13}$ We gathered published papers from these three digital sources between the years of 2013 and 2017.

Step 2: Papers Collection. To reduce bias in the context of the study, we only collected technical papers. Papers which did not meet our criteria (i.e shorter-than-10-page papers, editorials, panels, poster sessions, and opinions) were excluded. As depicted in Fig. 6, by applying our criteria, we sourced 3,057 papers in total from the three digital sources in a 5-year time span.

Step 3: Search String Definition and Execution. In this step, we formulated search keywords to filter the targeted papers into more specific works that use the git diff command. We defined three specific search terms related to the command, namely git, $\log$ and diff. Papers that contained one of three words with an exact match without affixes or suffixes (e.g. github, blog, logarithm, logging, different, difficult etc.) were collected. Since we only focus on the study that used diff command in git repositories, papers that do not exactly mention at least one of the three keywords are excluded despite they use other terms such as differencing which might indicates the implementation of the other diff tools. The command git log was also targeted because this command can produce diff with specific options. By using these three search terms, all papers extracted from the databases were then manually scanned in full text. Consequently, only published works containing these three search strings were included. As a result of Step 3, we were able to identify 137 papers.

Step 4: Full Text Reading. To ensure the collected previous studies are relevant to our objectives, we then performed a full text reading of the papers. This process was undertaken by the first and the second authors to avoid obscurity and to separate the primary

\footnotetext{
${ }^{12} \mathrm{https}: / /$ www.scimagojr.com/

${ }^{13} \mathrm{http}: / /$ www.core.edu.au/conference-portal/2018-conference-rankings- 1
} 


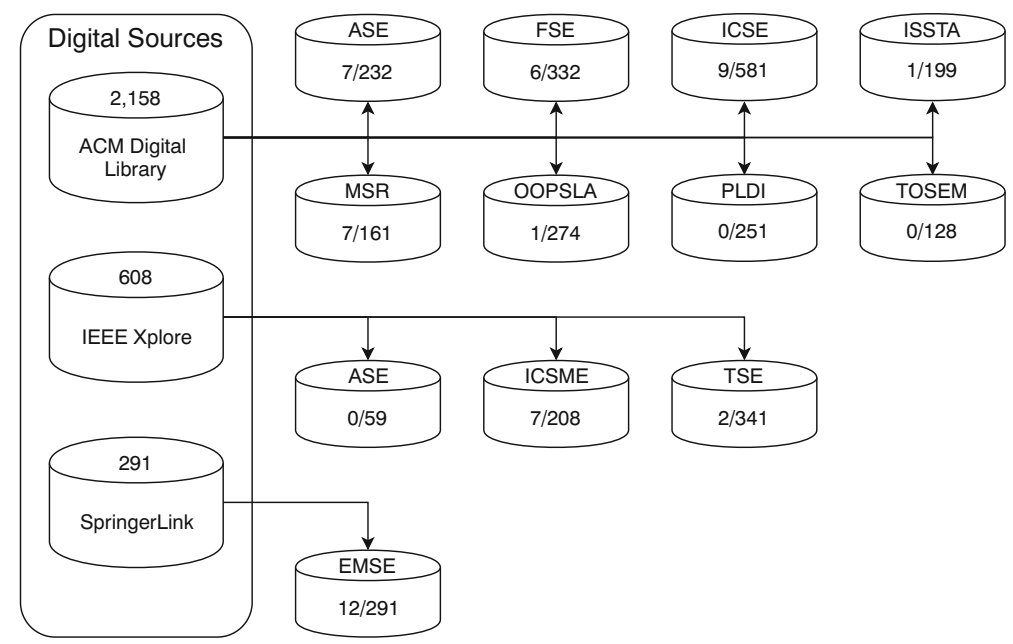

Fig. 6 Number of collected papers from each source

studies more exhaustively based on their contents. We applied the inclusive and exclusive criteria to the full paper which is described in Table 2. Papers that fit the inclusive criteria were kept for further processing while other papers that met the exclusive criteria were excluded from the study. After this step, we had 52 papers.

\subsection{Results of the Mapping}

Figure 7 indicates the distribution of the number of papers in each journal and conference in the last 5 years. As can be seen in the heat map, all journals and conference proceedings published the works related to the git diff command application in at least one paper in 5 years except for the PLDI and TOSEM. Most papers that applied the git diff command are published on EMSE especially in 2017, accounting for 6 papers.

\subsubsection{Which Diff Algorithm Is Used?}

Out of the 52 primary studies,we identified the application of different diff algorithms in the command in extracting the changes. Of particular note is that even though most instructions applied different options in the use of the git command to extract the required data, none of the previous selected works considered different diff algorithms. This shows that all of the collected studies used Myers as the default algorithm.

Table 2 Inclusive and exclusive criteria

\begin{tabular}{ll}
\hline Group & Criteria \\
\hline Inclusive & $\begin{array}{l}\text { Paper mentions the git command. } \\
\text { Paper applies the git command to extract the data from Git repositories for further } \\
\text { analyses in support of their works. }\end{array}$
\end{tabular}

Exclusive $\quad$ Paper does not use the git command as part of their studies. For example, git diff is used only for motivating examples. 


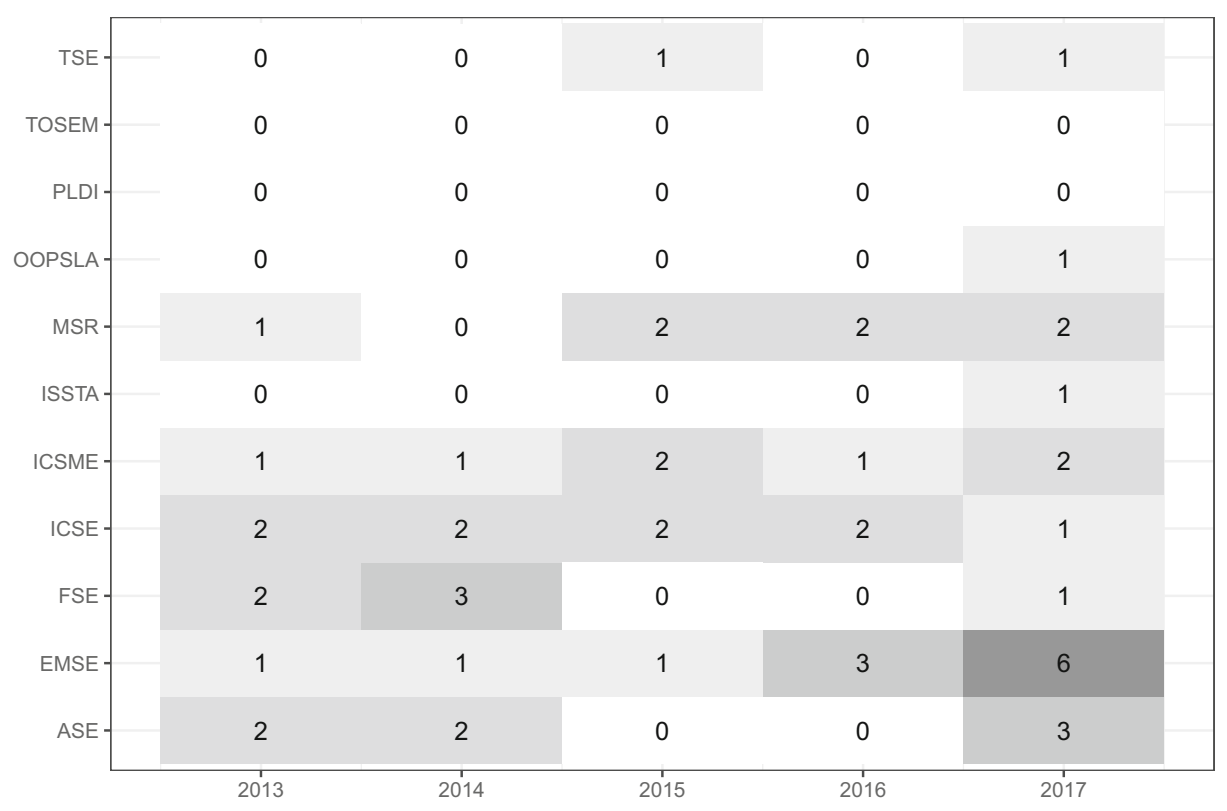

Fig. 7 Number of papers per journals and conferences between 2013 and 2017

\subsubsection{What Kind of Software Artifact Is Analyzed?}

To understand the components that were extracted using the git command in the previous studies, two main focuses emerged as our parameters to classify the documents; namely, code changes and license changes as depicted in Fig. 8. As can be seen in the figure, code changes were prominently the focus for researchers in extracting software repositories using the git command over five years. Thus, in our comparisons we analyze code changes extracted from the data source.

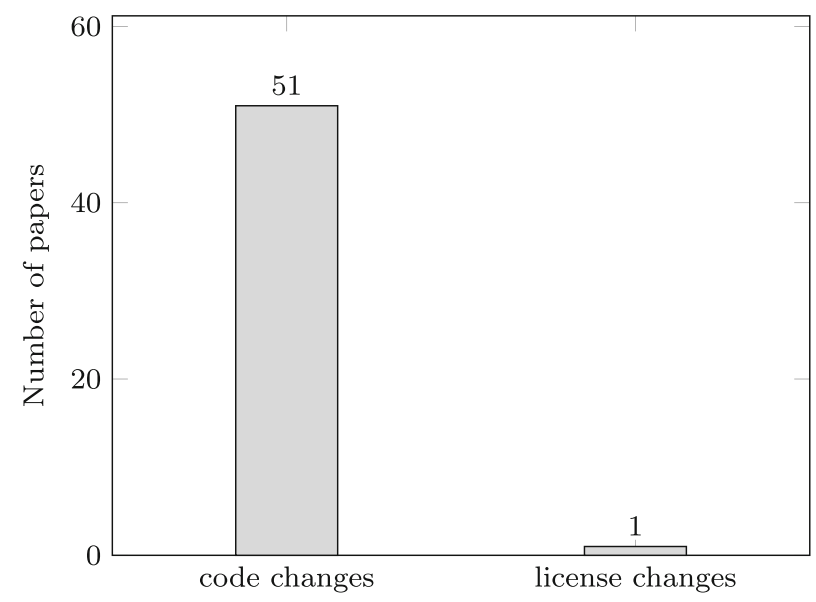

Fig. 8 Number of papers based on parameter searched using git command 


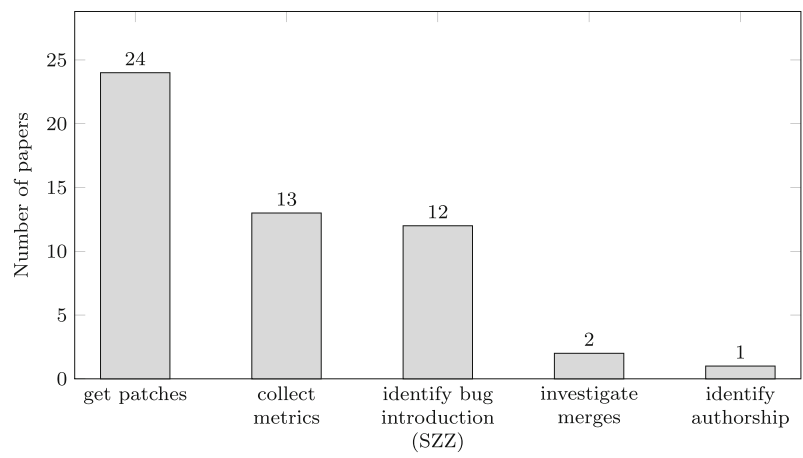

Fig. 9 Number of papers classified with the purpose of using the git command

\subsubsection{What Are Purposes of Using Diff?}

By reading the papers manually, we summarized the purposes from the extraction of software development records and grouped them into five categories, as can be seen in Fig. 9.

From the figure, we see that the most common purposes is to get patches, amounting to as many as 24 studies, followed by collecting metrics and identifying bug-introductions, which covered 13 and 12 studies, respectively. A few studies addressed merges investigation and authorship identification. This finding motivated us to carry out a further investigation of the impact of different diff algorithms in the extraction of the added and deleted lines for metrics collection, bug-introducing change identification, and getting the patches.

\subsubsection{Where Does the Data Source Come From?}

Our intention is to provide a comprehensive understanding of the different outcomes generated by different diff algorithms; thus, we need to run a set of tests of the algorithms' implementations in the git diff command. From the result of our dataset classification, open source software (OSS) is found to be dominated as the data source over the industrial type as illustrated in Fig. 10. Therefore, we mine the data from OSS projects to support our comparisons.

\subsection{Summary}

The survey results of the usage of the git diff command confirm that the previous studies conducted between 2013 and 2017 did not use various diff algorithms to extract

Fig. 10 Distribution of the type of data sources used in prior studies

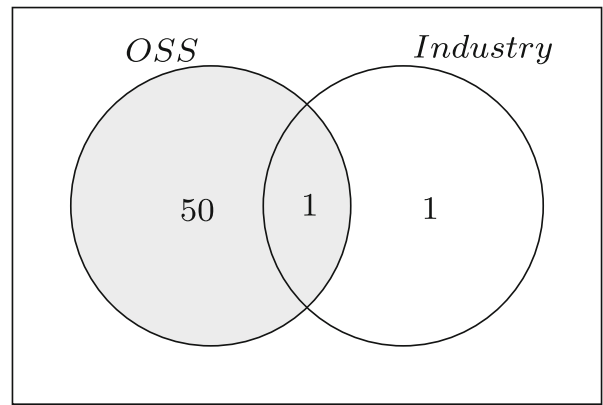


the differences between the first and the second versions of the same file. In mining the diff lists, they applied the standard commands using a default diff algorithm with some additional options, but without considering various diff algorithms. We also found that the information most sought after in prior studies was code changes in open source projects. The code changes were mostly utilized to thoroughly investigate counting the number of line changes and to record them in the form of metrics, locating the origin of a bug using a specific method (i.e. SZZ algorithm), and analyzing the patches. The results of these types of analyses obviously rely on the diff records produced by an applied diff algorithm in the git diff commands. Thus, different diff algorithms in extracting the line of code changes might differentiate the final result of a study and the conclusion of the description as well.

\section{Overview of Comparisons and Research Questions}

The findings from our systematic mapping revealed the three most common purposes for using the git diff command. This encouraged us to undertake comparison analyses between the Myers and Histogram algorithms in three applications: metrics, the SZZ algorithm, and patches. Our intention is to investigate the level of differences between the two diff algorithms used in these three applications and their possibility of affecting the result of studies. To achieve these goals, we address the following research questions:

\section{$\mathbf{R Q}_{1} \quad$ Can the values of diff-related metrics become different because of different diff algorithms?}

For metrics (Section 6), equal and unequal changed lines in the files identified by the two diff algorithms were calculated based on two factors: the quantity and the position of the line of code. We then compared the quantity of the files that have the same and different added and deleted lines of code to understand the significance of the differences of both algorithms in providing the diff records.

$\mathbf{R Q}_{2} \quad$ Are the results of bug-introducing change identification different because of different diff algorithms?

The result of locating bug-introducing changes using the SZZ algorithms relies on the diff results. In Section 7, we applied the Myers and Histogram algorithms in the git diff command to know whether the diff lists affect the result of bug-introducing change identification.

$\mathbf{R Q}_{3} \quad$ Which diff algorithm is better in generating a good diff?

Lastly, we compared the quality of the identified patches manually. In Section 8, we investigate 377 changes, a statistically representative sample of the 21,590 changes identified in the above two comparisons.

In our three comparisons, to extract the changes, we apply the git command: git diff -w --ignore-blank-lines --diff-algorithm=<algorithm> $<$ parentcommit ID $><$ commit ID $>--<$ filename $>$. We use the same options $-\mathrm{w}$ and --ignore-blank-lines to ignore whitespace and the changes whose lines are all blank. The use of various options is common according to the purposes to what extent the diff command generates the code changes. However, since our focus is comparing Myers and Histogram as the diff algorithm that can be used at the same circumstances, we do not consider to investigate the impact of other options. 


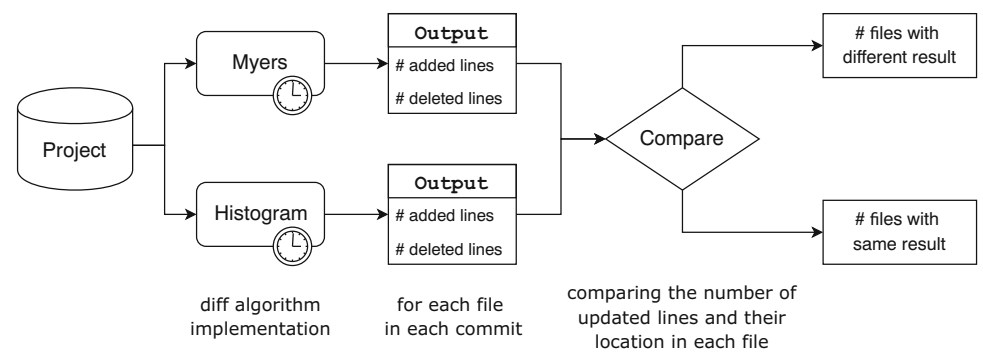

Fig. 11 Overview of the metrics collection procedure

\section{Comparison: Metrics $\left(\mathrm{RQ}_{1}\right)$}

$\mathbf{R Q}_{1}$ : Can the values of diff-related metrics become different because of different dif $f$ algorithms?

\subsection{Analysis Design}

As illustrated in Fig. 11, we investigate the following two basic diff-related metrics with two diff algorithms: Myers and Histogram.

NLA The number of added lines in a file.

NLD The number of deleted lines in a file.

For our empirical analysis, we collected the Git repositories of 14 projects used in the previous study (Rausch et al. 2017), which are identified in our systematic mapping as a study utilizing git for collecting metrics. The targeted 14 projects are OSS that employ Continuous Integration (CI) and are written in Java. The descriptions of the projects and the number of commits in the master branches are shown in Table 3.

We investigated all modified files in all commits in the master branches. To extract the NLA and NLD from the file, we implement the git command: git diff -w --ignore-blank-lines --diff-algorithm=<algorithm> <parent commit ID $>$ commit ID $>-<$ filename $>$. We considered the results the same if the values of both NLA and NLD were the same with the two algorithms; otherwise, the results were considered different. However, several software engineering tasks that rely on such metrics do not consider the position of the added and deleted lines, where different position of the changed lines can be occurred by chance despite the same metrics value. We conjecture that different number and position of changed lines can have different impact on empirical studies. Thus, we investigated the disagreement of the identified change locations separately. If the positions of each changed line of code were the same, we considered the results the same; otherwise, the results were considered different. File-level and commit-level results are discussed to see how the different results can appear in a different granularity.

\subsection{Results}

Table 4 summarizes the result from the comparison between two diff algorithms in 14 projects. From the total number of modified files identified by both algorithms, we counted 
Table 3 Targeted 14 open-source Java projects following the previous study (Rausch et al. 2017)

\begin{tabular}{llr}
\hline Project name & Description & \#Commits \\
\hline Apache Storm & Distributed realtime computation system & 9,317 \\
Butterknife & View binding library for Android & 836 \\
Crate & Distributed SQL database & 8,646 \\
Hystrix & Interactions controller library between distributed systems & 2,106 \\
JabRef & Reference manager application that uses BibTex & 11,940 \\
jcabi-github & Object oriented wrapper of GitHub API & 2,521 \\
Openmicroscopy & Application to store biological microscopy light data & 46,543 \\
& in a standard format & \\
Presto & Distributed SQL query engine for big data & 13,561 \\
RxAndroid & RxJava bindings for Android & 461 \\
SpongeAPI & A minecraft plugin API & 2,479 \\
Spring Boot & A framework to create Java applications & 17,087 \\
Square OkHttp & An HTTP+HTTP/2 client for Android and Java applications & 3,171 \\
Square Retrofit & An open source library to make HTTP communication simpler & 1,576 \\
WordPress-Android & WordPress for Android OS & 30,295 \\
\hline
\end{tabular}

the quantity of files in each commit that have same or different number values of NLA and NLD metrics. Similarity, the number of same and different results in changed locations are shown in the table.

We see that the percentages of different metric values are between $0.8 \%$ and $6.2 \%$. Considering the different results in locations of changes, ranging from $1.4 \%$ to $7.6 \%$, we found

Table 4 Total number of files that have the same and different values in metrics (NLA and NLD) and the position of changes

\begin{tabular}{|c|c|c|c|c|c|c|c|c|c|}
\hline \multirow{3}{*}{$\begin{array}{l}\text { Project } \\
\text { Apache Storm }\end{array}$} & \multirow{3}{*}{ 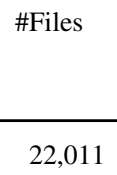 } & \multicolumn{4}{|c|}{ Metrics (NLA and NLD) } & \multicolumn{4}{|c|}{ Locations of Changes } \\
\hline & & \multicolumn{2}{|l|}{ \#Same \% } & \multicolumn{2}{|c|}{ \#Different \% } & \multicolumn{2}{|l|}{ \#Same \% } & \multicolumn{2}{|c|}{ \#Different $\%$} \\
\hline & & 21,278 & $96.7 \%$ & 733 & $3.3 \%$ & 20,979 & $95.3 \%$ & 1,032 & $4.7 \%$ \\
\hline Butterknife & 1,873 & 1,804 & $96.3 \%$ & 69 & $3.7 \%$ & 1,774 & $94.7 \%$ & 99 & $5.3 \%$ \\
\hline Crate & 44,463 & 43,522 & $97.9 \%$ & 941 & $2.1 \%$ & 42,723 & $96.1 \%$ & 1,740 & $3.9 \%$ \\
\hline Hystrix & 3,310 & 3,192 & $96.4 \%$ & 118 & $3.6 \%$ & 3,097 & $93.6 \%$ & 213 & $6.4 \%$ \\
\hline JabRef & 55,988 & 54,375 & $97.1 \%$ & 1,613 & $2.9 \%$ & 53,609 & $95.7 \%$ & 2,379 & $4.3 \%$ \\
\hline jcabi-github & 6,218 & 6,170 & $99.2 \%$ & 48 & $0.8 \%$ & 6,131 & $98.6 \%$ & 87 & $1.4 \%$ \\
\hline Openmicroscopy & 118,349 & 115,126 & $97.3 \%$ & 3,223 & $2.7 \%$ & 112,548 & $95.1 \%$ & 5,801 & $4.9 \%$ \\
\hline Presto & 73,572 & 72,471 & $98.5 \%$ & 1,101 & $1.5 \%$ & 71,455 & $97.1 \%$ & 2,117 & $2.9 \%$ \\
\hline RxAndroid & 627 & 613 & $97.8 \%$ & 14 & $2.2 \%$ & 603 & $96.2 \%$ & 24 & $3.8 \%$ \\
\hline SpongeAPI & 10,757 & 10,584 & $98.4 \%$ & 173 & $1.6 \%$ & 10,395 & $96.6 \%$ & 362 & $3.4 \%$ \\
\hline Spring Boot & 62,137 & 60,805 & $97.9 \%$ & 1,332 & $2.1 \%$ & 60,214 & $96.9 \%$ & 1,923 & $3.1 \%$ \\
\hline Square OkHttp & 7,345 & 7,206 & $98.1 \%$ & 139 & $1.9 \%$ & 7,108 & $96.8 \%$ & 237 & $3.2 \%$ \\
\hline Square Retrofit & 3,473 & 3,394 & $97.7 \%$ & 79 & $2.3 \%$ & 3,351 & $96.5 \%$ & 122 & $3.5 \%$ \\
\hline WordPress Android & 58,188 & 54,555 & $93.8 \%$ & 3,633 & $6.2 \%$ & 53,789 & $92.4 \%$ & 4,399 & $7.6 \%$ \\
\hline
\end{tabular}


that quite a few portions of the metric values are same even though the identified locations are different.

To further explore of the disagreements between Myers and Histogram, we calculated the number of commits influenced by the different number of code changes and the locations in the diff output of files. In each project, we counted the sum of files that have the same and different quantity and the position of lines inserted and removed from each commit across the project. A single commit may contain more than one modified file. If a commit recorded at least one file having unequal changed lines of code either in their number or their location, we classified this commit as 'different'. On the other hand, if all files in a commit had identical changed lines, we categorized the commit in the 'same' class. In this process, we only notify the files that have an unequal number and location of the lines of code.

Our results show that several changed files impacted by the changed lines have similar commits. We grouped the same commits from these several files that contain different changed lines of code into a single commit. We then summarized the percentage of commits that have a different number and position of the changed lines of code resulting from the usage of the Myers and Histogram algorithms in the git diff command as described in Table 5.

In general, our comparisons revealed that the data extraction using two diff algorithms in the command produced identical diff lists for most files in all commits. However, even though the output has been dominated by the same results for each file in a commit, the diff output from the Myers and Histogram recorded several files that have different added and deleted lines. These disagreements impacted the dissimilar number of commits that have files containing changed lines of code. The level of differences in the number of commits influenced by the amount of lines of code are adequately high, ranging from $1.7 \%$ to $8.2 \%$, while the unequal location of lines affects the level of differences in the quantity of commits from $2.8 \%$ to $13.9 \%$.

Table 5 The number of commits that contain a different number and the position of added and deleted lines of code in a file

\begin{tabular}{|c|c|c|c|c|c|}
\hline \multirow[t]{2}{*}{ Project } & \multirow[t]{2}{*}{ \#Commits } & \multicolumn{2}{|c|}{ Metrics (NLA and NLD) } & \multicolumn{2}{|c|}{ Locations of Changes } \\
\hline & & \#Different & $\%$ & \#Different & $\%$ \\
\hline Apache Storm & 9,317 & 395 & $4.2 \%$ & 587 & $6.3 \%$ \\
\hline Butterknife & 836 & 34 & $4.1 \%$ & 48 & $5.7 \%$ \\
\hline Crate & 8,646 & 707 & $8.2 \%$ & 1,202 & $13.9 \%$ \\
\hline Hystrix & 2,106 & 87 & $4.1 \%$ & 160 & $7.6 \%$ \\
\hline JabRef & 11,940 & 881 & $7.4 \%$ & 1,317 & $11.0 \%$ \\
\hline jcabi-github & 2,521 & 42 & $1.7 \%$ & 70 & $2.8 \%$ \\
\hline Openmicroscopy & 46,543 & 2,340 & $5.0 \%$ & 3,674 & $7.9 \%$ \\
\hline Presto & 13,561 & 816 & $6.0 \%$ & 1,423 & $10.5 \%$ \\
\hline RxAndroid & 461 & 11 & $2.4 \%$ & 18 & $3.9 \%$ \\
\hline SpongeAPI & 2,479 & 128 & $5.2 \%$ & 235 & $9.5 \%$ \\
\hline Spring Boot & 17,087 & 954 & $5.6 \%$ & 1,447 & $8.5 \%$ \\
\hline Square OkHttp & 3,171 & 106 & $3.3 \%$ & 181 & $5.7 \%$ \\
\hline Square Retrofit & 1,576 & 61 & $3.9 \%$ & 90 & $5.7 \%$ \\
\hline WordPress Android & 30,295 & 2,108 & $7.0 \%$ & 3,050 & $10.1 \%$ \\
\hline
\end{tabular}




\subsection{Summary}

The finding from the metrics comparison provides clear evidence that the use of multiforms of diff algorithms might differentiate the diff lists. Since the metrics are insensitive to differences in change locations, the same values can be obtained even if identified change locations are different. However, we see that different metric values were obtained from $0.8 \%$ to $6.2 \%$ in the file-level and $1.7 \%$ to $8.2 \%$ in the commit-level. These differences can have impacts on studies using diff-related metrics.

\section{Comparison: $\mathrm{SZZ}$ Algorithm $\left(\mathbf{R Q}_{2}\right)$}

$\mathbf{R Q}_{2}$ : Are the results of bug-introducing change identification different because of different diff algorithms?

\subsection{SZZ Algorithm}

The SZZ algorithm proposed by Śliwerski et al. (2005) is an approach to identify bugintroducing changes. The SZZ uses a bug-tracking system (e.g. Bugzilla) as the reference to link archived versions of a software (e.g. CVS). Figure 12 depicts the basic idea of the SZZ algorithm.

The SZZ algorithm first identifies bug-fixing commits by searching bug report identity numbers (bug ID) in log messages, which have been written by developers when they fix bugs. The commit ID of this bug-fixing commit is subsequently used to track the previous commit (parent commit). The code changes are extracted by applying diff to find the differences between the older version of a file in the parent-commit and the newer version of the same file in the bug-fix commit. The identified deleted lines are considered to be candidates of bug-related lines. To identify bug-introducing commits, cvs annotate command is used to investigate when lines are added. Among the candidates of bug-related lines, lines that have been created before the bug reporting time are considered to be validated bug-related lines. The commits that introduced those validated bug-related lines are identified as bug-introducing commits.

\subsection{Analysis design}

Figure 13 describes the validation process of our analysis. For our empirical analysis, we studied 10 open source Apache projects used in the previous study (da Costa et al. 2017),

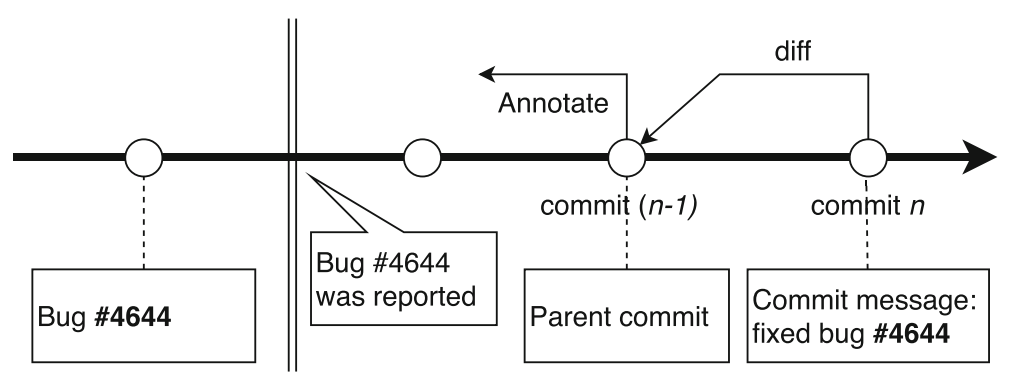

Fig. 12 SZZ: Locating bug-introducing changes 


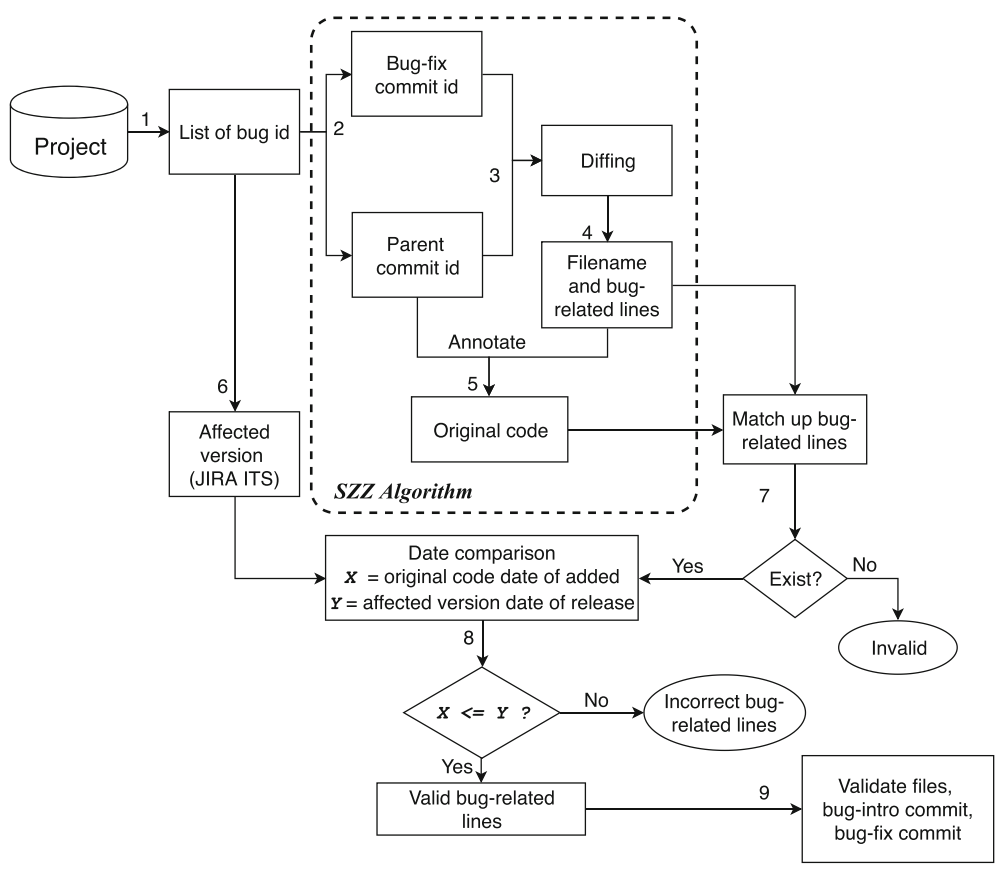

Fig. 13 Overview of the validation process of bug-introducing commit

which is identified in our systematic mapping as a study utilizing Git for identifying bug introduction using the SZZ algorithm. The descriptions of projects and the number of commits in the master branches are shown in Table 6. We analyzed the impact of using different diff algorithms on the original SZZ algorithm. We studied the disagreement between the Myers and Histogram in the results of the SZZ algorithm based on diff.

Table 6 Overview of the 10 studied Apache projects

\begin{tabular}{llr}
\hline Project Name & Description & \# Commits \\
\hline ActiveMQ & Message broker and Java Message Service client & 9,962 \\
Camel & A framework to create routing and mediation rules in & 32,124 \\
& various domain specific languages & 8,184 \\
Derby & Relational database implemented in Java & 13,137 \\
Geronimo & Open source application server compatible with Java EE & 10,509 \\
Hadoop Common & Collection of common utilities and libraries that sup- & 15,091 \\
HBase & port other Hadoop modules & 3,959 \\
Mahout & A distributed big data store for the Hadoop database & 4,864 \\
OpenJPA & A library to generate the implementation of dis- & 3,154 \\
Pig & tributed or scalable machine learning algorithms & 16,253 \\
Tuscany & The implementation of the Java Persistence API & High-level mechanism for the parallel programming \\
\hline
\end{tabular}


First, bug report IDs in the commit messages are searched with specific keywords (i.e. "bug", "fix", "defect", and "patch" (Śliwerski et al. 2005)), then the identified commits are marked as candidates of bug-fixing commits. In each candidate bug-fixing commit, we focus on the modified files. The two diff algorithms are used to identify deleted lines using the command: git diff -w --ignore-blank-lines--diff-algorithm= <algorithm> <parent commit ID> <bug-fix candidate commit ID > - $<\mathrm{filename}>$. By fetching files in the parent commit ID, we subsequently applied the git blame command (similar to cvs annotate) to locate the origin of the deleted lines. Those deleted lines are considered to be candidates of bug-related lines.

Similar to the procedure of da Costa et al. (2017), the next step is to find the affected software versions of a bug. We extract bug reports and their affected versions from the JIRA issue tracking system. ${ }^{14}$ If a single bug ID affects more than one version, the earliest version is chosen since the SZZ algorithm targets the initial appearance of a bug. From the collection of affected-versions, we compare the dates of the introduction of the candidates of bug-related lines with the release dates of the versions. If the release dates of the affected versions are later than the dates of the introduction of the candidates of bug-related lines, we classified them as valid bug-related lines; otherwise, we classified them as invalid.

With these sets of valid bug-related lines, we validate bug-introducing commits, bugrelated files and bug-fixing commits. The validation processes are performed in the opposite direction with the above procedure. A valid bug-introducing commit is a commit that initially adds valid bug-related lines. Files containing bug-related lines are considered to be valid bug-related files. From the candidates of bug-fixing commits, if there is at least one valid associated bug-introducing commit, we consider the candidate bug-fixing commit to be valid, otherwise invalid.

\subsection{Results}

Table 7 presents the outputs of the Myers and Histogram algorithms in the number of valid bug-related lines, files, bug-introducing commits, and bug-fix commits. Two algorithms produced a different number of valid bug-related lines in all 10 projects, which then led to the different number of files, bug-introducing commits, and bug-fix commits.

Similar to the analysis of metrics in Section 6, differences in the quantities of changes are relatively small or the same for some projects, because of the insensitivity of change locations.

Since investigating the locations of bug introduction is also important, we perform a comparison of files that have the same and different locations of bug-related lines. Table 8 shows this result. It can be seen that the total number of files that have a different location of the changed code is high in each project, ranging from $2.4 \%$ to $6.6 \%$. This means that some files can contain suspicious bug-related lines, only because of different algorithms.

Bringing these data into further analysis, we then summarized the number of valid bugfixing commits. As shown in Fig. 14, all studied projects have a different number of valid bug-fixing commits caused by the different positions of valid bug-related lines resulting from the Myers and Histogram. The percentage of the different results are between $6.0 \%$ and $13.3 \%$, or $9.7 \%$ on average. This analysis found evidence that nearly $10 \%$ of bugfixing commits do not guarantee success in locating bug-introducing changes since some

\footnotetext{
${ }^{14}$ https://www.atlassian.com/software/jira (April 2018)
} 
Table 7 Summary of valid bug-related lines, valid files, valid bug-introducing commits, and valid bug-fix commits resulting from Myers and Histogram

\begin{tabular}{|c|c|c|c|c|c|c|c|c|}
\hline \multirow[t]{2}{*}{ Project } & \multicolumn{2}{|c|}{$\begin{array}{l}\text { \#valid bug- } \\
\text { related lines }\end{array}$} & \multicolumn{2}{|c|}{ \#valid files } & \multicolumn{2}{|c|}{$\begin{array}{l}\text { \#valid bug- } \\
\text { intro commits }\end{array}$} & \multicolumn{2}{|c|}{$\begin{array}{l}\text { \#valid bug- } \\
\text { fix commits }\end{array}$} \\
\hline & M & $\mathrm{H}$ & M & $\mathrm{H}$ & M & $\mathrm{H}$ & M & $\mathrm{H}$ \\
\hline ActiveMQ & 10,671 & 10,846 & 1,566 & 1,565 & 1,015 & 1,016 & 614 & 613 \\
\hline Camel & 12,525 & 12,626 & 2,377 & 2,374 & 1,514 & 1,516 & 716 & 712 \\
\hline Derby & 130,861 & 131,031 & 4,372 & 4,373 & 1,178 & 1,180 & 1,038 & 1,039 \\
\hline Geronimo & 29,543 & 29,743 & 2,448 & 2,448 & 1,282 & 1,277 & 462 & 462 \\
\hline Hadoop Common & 15,053 & 15,285 & 805 & 805 & 546 & 550 & 318 & 318 \\
\hline HBase & 37,558 & 37,291 & 2,083 & 2,079 & 1,480 & 1,481 & 669 & 668 \\
\hline Mahout & 1,542 & 1,548 & 182 & 182 & 145 & 144 & 44 & 44 \\
\hline OpenJPA & 5,160 & 5,204 & 794 & 794 & 370 & 370 & 365 & 366 \\
\hline Pig & 1,789 & 1,787 & 205 & 206 & 187 & 187 & 80 & 80 \\
\hline Tuscany & 750 & 781 & 46 & 46 & 34 & 36 & 16 & 16 \\
\hline
\end{tabular}

$M=$ Myers

$H=$ Histogram

deleted lines that were suspected as the candidate bug-introducing changes are different if we applied different diff algorithms in the git diff command. This is because a valid bug-related line in a file has the possibility of being identified by a particular diff algorithm, but it remains undetected while using the other diff algorithms.

\subsection{Summary}

The results from the SZZ algorithm confirm that different diff algorithms possibly generate different results, from $6.0 \%$ and $13.3 \%$ in the total of the identified bug-fix commits. The Myers and Histogram sometimes produced a different number and location of the deleted lines (bug-related lines) in several files. These differences certainly affect the number of

Table 8 Total number of files that have the same and different positions of valid bug-related lines in all valid bug-fix commits

\begin{tabular}{lrrrr}
\hline Project & \#Same & \#Different & $\%$ & Total \\
\hline ActiveMQ & 1,464 & 103 & $6.6 \%$ & 1,567 \\
Camel & 2,315 & 63 & $2.7 \%$ & 2,378 \\
Derby & 4,198 & 175 & $4.0 \%$ & 4,373 \\
Geronimo & 2,318 & 130 & $5.3 \%$ & 2,448 \\
Hadoop Common & 777 & 28 & $3.5 \%$ & 805 \\
HBase & 1,973 & 110 & $5.3 \%$ & 2,083 \\
Mahout & 171 & 31 & $6.0 \%$ & 182 \\
OpenJPA & 764 & 5 & $3.9 \%$ & 795 \\
Pig & 201 & 3 & $2.4 \%$ & 206 \\
Tuscany & 43 & $6.5 \%$ & 46 \\
\hline
\end{tabular}




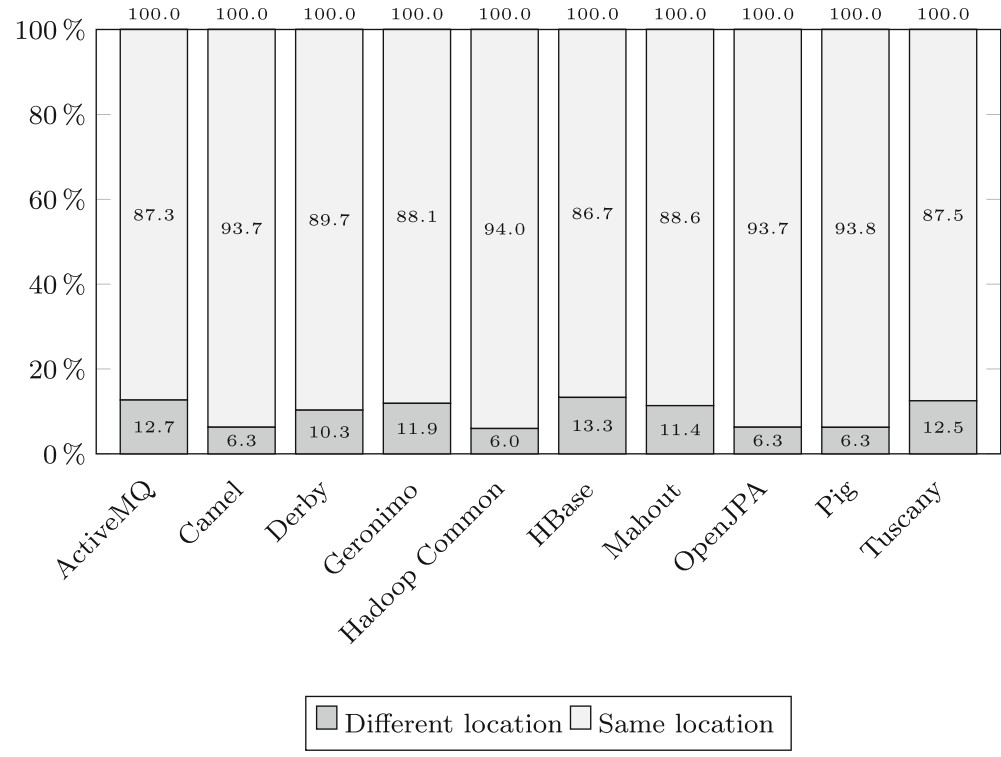

Fig. 14 The percentage of valid bug-fixing commits that have the same and different positions of valid bug-related lines

disagreement files that have the bug-related lines, the amount of bug-introducing commits, and the bug-fixing commits that actually have the bug-contained files. Therefore, the comparison result indicates that several prior studies that had used the SZZ algorithm to locate bugs have the possibility of producing inaccurate analyses.

\section{Comparison: Patches $\left(\mathbf{R Q}_{3}\right)$}

$\mathbf{R Q}_{3}$ : Which diff algorithm is better in generating a good diff?

\subsection{Analysis Design}

From the previous two comparisons, we showed that different diff algorithms can have different results of metrics collection and bug-introduction identification (SZZ algorithm). Computationally, both diff algorithms are correct in textual differencing. However, the diff outputs are sometimes different due to different diff algorithms. The diff results might show different change region with a contiguous list of deleted and added lines that is called as a change hunk (Ray et al, 2015). We expect that a set of changing operations

Table 9 Targeted files that have different locations in identified lines with two diff algorithms

\begin{tabular}{llr}
\hline Project Group & Type of identified line & \#Files \\
\hline CI-Java Projects (Section 6) & Added and deleted lines & 20,535 \\
Apache Projects (Section 7) & Deleted lines & 1,055 \\
Total & & 21,590 \\
\hline
\end{tabular}


Table 10 Description of the diff assessment

\begin{tabular}{ll}
\hline Result & Condition \\
\hline Histogram & The output of Histogram is better. \\
Myers & The output of Myers is better. \\
Same & Both outputs are same level. One algorithm is not better than the other.
\end{tabular}

done by developers can be represented by change hunks. However, the identification of the change hunks can be inappropriate. In our investigation, this issue could not be identified automatedly. Thus, we analyze the quality of diff manually.

To judge the quality of the diff algorithms, we define "better" if the algorithms meet our two criteria: (i) it detects the unmodified lines appropriately that should not be identified as changed lines, and (ii) it shows the changed lines more systematically (Kim et al. 2013). The sequences of the added and deleted lines of code are expected to be closer to what developers did to the code. If the code elements change together, they are shown explicitly as group systematic changes or report their common structural characteristics.

For this analysis, we used the same dataset that had been used in Sections 6 and 7, shown in Table 9. From the CI-Java projects, we considered all modified files in all commit IDs to be targeted, while of the Apache projects, files changed in all bug-fix commit candidates are targeted. We applied the same command as the other two comparisons: git diff -w --ignore-blank-lines --diff-algorithm=<algorithm> <parent commit ID> <commit ID> - - <filename s to generate the diff output from Myers and Histogram. In each project of the first group, we analyzed the files that have different locations of the inserted and removed lines from the execution of the two diff strategies. While in the second group, only the files that have a different location of the deleted lines were analyzed.

We divided the comparison into two categories: (i) in-code diff and (ii) in-non-code diff. The first category of diff means the different diff lists generated by both algorithms are lines of code or a block of code in a source code file. Otherwise, the second diff implies the disagreement between these two algorithms are other than a line of code, for example a change of comments, or a change in a non-code file, such as a modification in a text file.

Qualitative analysis between the two diff algorithms was performed manually by the first two authors in multiple steps. Initially, the first author made a list of all files from the two project groups. From this list, the sample size of files was counted using the tool provided in a survey system ${ }^{15}$ to statistically represent sample from files in each project, so that the conclusions about the quality of the diff algorithm would generalize to all files in all projects with a confidence level of $95 \%$ and a confidence interval of 5 . As can be seen in Table 9, the total number of files summarized from all project groups is 21,590 . From this population, we selected random samples of 377 files.

In the second step, we conducted a manual comparison between two diff outputs produced by Myers and Histogram algorithms from all files in the sample. The first two authors of this paper were involved to independently annotate the diff outputs that makes the result is expected to be more reliable. To specify the comparison result between two diff algorithms, we generated three categories as described in Table 10. We assign Histogram to the comparison results if the diff outputs produced by Histogram algorithm show the

${ }^{15}$ https://www.surveysystem.com/sscalc.htm 
Table 11 Frequency of comparison result in the sample data

\begin{tabular}{lcccc}
\hline Result & in-Code diff & \multicolumn{3}{c}{ in-Non-Code diff } \\
\hline Histogram & 152 & $(62.6 \%)$ & 18 & $(13.4 \%)$ \\
Myers & 41 & $(16.9 \%)$ & 20 & $(14.9 \%)$ \\
Same & 50 & $(20.6 \%)$ & 96 & $(71.6 \%)$ \\
Sum & 243 & $(100 \%)$ & 134 & $(100 \%)$ \\
\hline
\end{tabular}

unmodified lines more appropriately and provide better group systematic changes to show the lines were changed together compared with the Myers. If the results produced by Myers provide more appropriate unchanged contexts and show the group changes more systematically compared with the Histogram's diff, we labeled them as Myers. While if the diff outputs produced by one algorithm are not better than the other, then we mark them the Same. The comparison results between two authors from 377 files were subsequently computed to find the kappa agreement. ${ }^{16}$ We obtained $70.82 \%$, which is categorized into 'substantial agreement' (Viera and Garrett 2005). This means, the statistic result of our manual study is acceptable.

\subsection{Results}

Table 11 shows how well both diff algorithms work in presenting the changes of code. It can be seen that Histogram outnumbered the other results in the in-Code diff category, which emphasizes that this algorithm is substantially better to differentiate the changes of code specifically.

Figure 15 shows how the Histogram algorithm provides better output of code changes compared with the Myers. We extracted the diff from the file AmqpMessage. java ${ }^{17}$ in commit $f 56$ ea 45 e 5 from the project of Active $M Q$. It is true that none of the algorithms are incorrect in describing changes. However, the Histogram algorithm provides a reasonable diff output better describing human change intention, as the if-statement is moved to a new method and a new method call is added. While from the result of Myers, it is not clear how developer changed the code. Lines that have not modified were identified as removed from the original positions (line 18 and 19) and added to the new positions (line 6 and 7).

This manual investigation also highlighted that the Myers and Histogram algorithms have almost the same ability to extract the diffs from non-code changes. As shown in Table 11, their percentages are nearly equals in the in-Non-Code diff (13.4\% files are better using the Histogram and $14.9 \%$ files are preferable using the Myers). This is even strengthened by the high percentage of both diff algorithms' application that resulted in the same quality for the same files (see the example in Fig. 16), which reached 71.6\%. This quantification reveals that we can use any of these algorithms to produce the diff from non-code changes. As shown in Fig. 16, both diff algorithms worked well to reveal the comment

\footnotetext{
${ }_{16}$ http://justusrandolph.net/kappa/

${ }^{17}$ activemq-amqp/src/test/java/org/apache/activemq/transport/amqp/client/ AmqpMessage.java - https:// github.com/apache/activemq/commit/f56ea45e58a17fa3aad46cbe8fc605ef4ffdbc81\#diff-5296b90814217d7 5e272e14834a09dca
} 


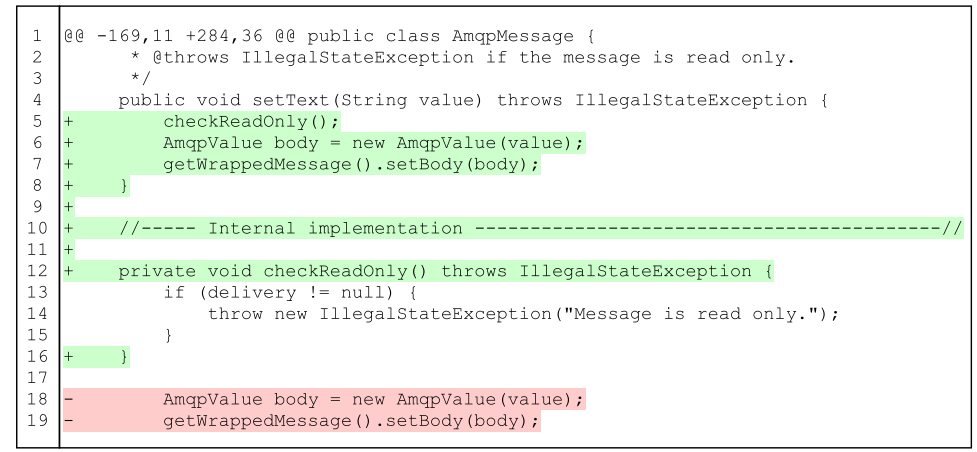

(a) diff output using Myers

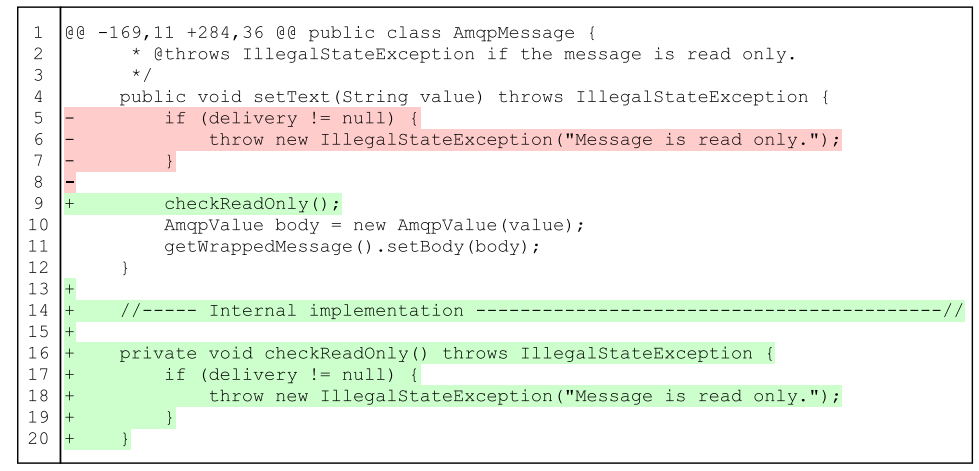

(b) diff output using Histogram

Fig. 15 Example of diff outputs generated by Myers and Histogram in extracting the code changes

changes from file ChannelMetadataLoader.java ${ }^{18}$ in commit e5924527fa of Openmicroscopy project since both lists are readable and understandable. The only differences between the two lists are the position of the initial added line and the matched line after the first inserted one. However, these disagreements did not change our interpretation about the modifications that occurred.

\subsection{Summary}

Due to the different procedures between Myers and Histogram in identifying the changed lines of code, they possibly generated different diff results. Our manual comparison found that their differences were the number of the changes, the order of the changed lines, or even the detected added and deleted code. They certainly affect the readability of the diff outputs, in other words, the quality of the diff results produced by the two diff algorithms were different. Importantly, our results provide evidence that Histogram frequently produced better diff results compared to Myers in extracting the differences in source code.

\footnotetext{
${ }^{18}$ SRC/org/openmicroscopy/shoola/agents/hiviewer/ChannelMetadataLoader.java - https:/github.com/ openmicroscopy/openmicroscopy/commit/e5924527fa467e117337b53a769503b6cc48e43f\#diff-94a26d7e2 56533ade03740d86aca1afe
} 


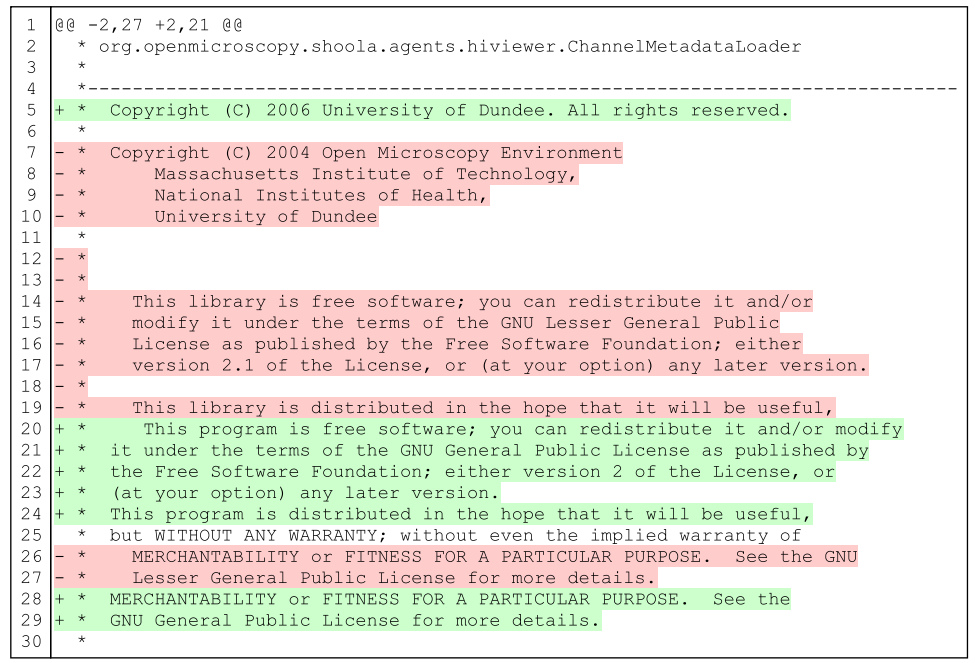

(a) diff output of comment using Myers

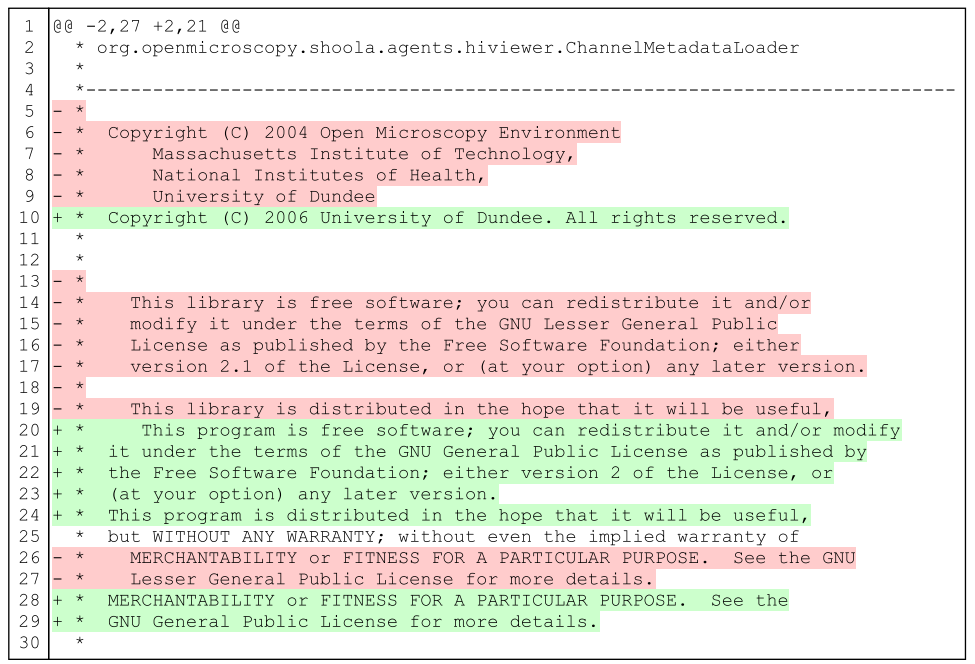

(b) diff output of comment using Histogram

Fig. 16 Example of diff lists generated by Myers and Histogram in extracting the non-code changes

\section{Discussions}

\subsection{Implication and Recommendation}

In this paper, we present a description of the impact of different diffs on the results of a study. In the example shown in Fig. 15, we can see both algorithms identify the changed lines of code from line \#169. Nevertheless, there are several differences in the identified changed lines shown in both diff outputs. 
The first difference is the number of the changed lines. From Fig. 15, we can see that the quantity of the detected changed lines are unequal. There are 11 changed lines discovered by the Myers, while the Histogram found 13 lines. In a study that aims to collect metrics from the code changes, considering different diff algorithms is important since it has an impact on the number of changes.

In software quality analysis, one key factor of process metrics used to measure the changes is the number of modified lines (NLA and NLD). For example, a work undertaken by Gousios et al. (2008) which proposed an approach to measure a software developer's contribution using diff records to compute the number of changed lines in a file. This quantity of the changed lines was then used to calculate the commit size of all affected files. Based on our metrics comparison, we found that $1.7 \%$ to $8.2 \%$ commits have different NLA and NLD due to different diff algorithms application. While our manual investigation shows that more than $60 \%$ diff outputs are better to extract using Histogram. Thus, if this study attempts to apply Histogram, it might affect around $1 \%$ to $4 \%$ different commit size. As a result, this will impact the measurement of software developer's contribution as well. Another study related to metrics analysis was conducted by Rausch et al. (2017). The authors investigated the complexity of changes that can impact software quality. The findings support that higher median values of NLA and NLD lead to an increase in build failures. The study also found that the high mean values of the number of modified files correlates to the failed builds. Based on the result from our metrics analysis, we found $0.8 \%$ to $6.2 \%$ files have different NLA and NLD. Therefore, if Histogram is applied in this study, this will influence around $0.5 \%$ to $3.5 \%$ of the modified files that correlates to the failed builds.

The second difference is the position of the changed lines. Figure 15 shows that the two diff algorithms detect the deleted lines differently. The Myers identifies one line of 'Assignment' and one line of a 'Method' call, whereas the Histogram specifies a block of 'if condition'. Related to SZZ application, both diff algorithms produce different deleted lines that are considered as the candidate of bug-introducing changes. Thus, the identified bug-related lines might be invalid due to different diff algorithms application that can lead to the failure of bug-introducing changes identification.

A study undertaken by da Costa et al. (2017) investigates the output of five SZZ procedures in discovering the bug-introducing changes. The study on 10 Apache projects analyzed the validity of bug-introducing changes. The validation process of bug-related lines used by the authors is similar to our study. It compares the release dates of the earliest affected software versions of a bug with the dates of the introduction of the candidates of bug-related lines. However, in our study, we enhanced the process to validate the other three parameters, that is, the bug-introducing commits that initially adds the valid bug-related lines, files containing valid bug-related lines, and bug-fixing commits that relates to valid bug-introducing commits. Our SZZ analysis shows that different diff algorithms application can have impact on the results of SZZ algorithm. We found $2.4 \%$ to $6.6 \%$ valid files have different location of valid bug-related lines. Since the Histogram is better in more than $60 \%$ diff outputs based on our manual analysis, therefore, if the study by da Costa et al. (2017) applies Histogram in the diff command, around 1.5\% to 4\% files might have different valid bug-related lines in their study results. The SZZ algorithm has also been studied by Rodriguez-Perez et al. (2018). The authors conducted a literature review of published articles that focus on the SZZ algorithm's functionality and its ability to be imitated. The similarity of this study to ours is investigating the changing impact due to the modification of SZZ algorithm. However, the study focus on the usability of the changing SZZ in the academic paper over time while our study analyze the impact of different diff algorithms application in the SZZ to study results. Without considering the version of SZZ used in 187 
previous studies collected by Rodriguez-Perez et al. (2018), we understand that SZZ is a widespread and well-known algorithm over a 10-year period. This bug identification algorithm was commonly used to investigate commit size ( $26 \%$ of the papers), line of code ( $15 \%$ of the papers), number of changes (12\% of the papers), number of affected files ( $8 \%$ of the papers), etc. As described in our SZZ analysis, diff algorithms also have an impact on SZZ. Thus, if the Histogram is applied in those 187 prior studies, it might affect the results of studies.

Our investigations on metrics and SZZ application provide evidences that different diff algorithms application in git command can have an impact on a study result. It is also acknowledged that the Histogram algorithm is substantially better than the Myers to produce the changed lines of code. Thus, we recommend to use the Histogram in git diff command to extract the changes from source code.

\subsection{Threats to Validity}

Threats to the construct validity appear in the mapping study and the SZZ application. In our mapping study, we selected only the papers that specifically mention the git commands. As a result, papers that had used git commands but do not mention it in the full text had been ignored, which can cause selection bias. Since different diff algorithms produce different results, we consider that papers should mention algorithm names of diff if the authors intentionally chose them. In the SZZ application, we used a small number of keywords to detect commit messages that describe fixing bugs. This limited our ability to extract all potential candidate bug-fixing commits. Even so, the commits that should not be identified as bug-fixing commits were also possible to be collected as long as they included the keywords in their log messages. However, since our focus is to investigate the level of differences of the diff lists produced by Myers and Histogram, the impact of the incorrect commits to the study result is small. Another threat to the construct validity is the definition of better for the diff algorithm. We consider good quality of the algorithm based on our two criteria, while many could have been considered. Different software engineering tasks may have different requirements for diff analysis. However, since our focus is expecting to recover the changing operations from the diff outputs, the impact of this issue is not significant.

Threats to the external validity emerge regarding the repository used in our experiments. Although we analyzed 24 OSS Java projects mined from Git repositories, we cannot generalize our study results to other open source projects nor industry.

To reduce the threats to reliability, we make our dataset publicly available. We provided lists of our collected files identified by the Myers and Histogram algorithms which were used in the three empirical analyses (see on GitHub ${ }^{19}$ ).

\section{Conclusion}

To understand the impact of using different diff algorithms, Myers and Histogram, we first clarified applications of diff by conducting a systematic mapping of papers published between 2013 and 2017. We then empirically analyzed the impact in three major applications: (i) code churn metrics, (ii) SZZ algorithm, and (iii) patches extraction.

\footnotetext{
${ }^{19}$ https://github.com/yusufsn/DifferentDiffAlgorithms
} 
Our quantitative analyses has shown that the different diff algorithms can report different amount of changed lines, identify different change locations. Our qualitative investigation revealed that Histogram is better for describing code changes. Since diff is the fundamental tool for various software engineering tasks, considering limitations and advantages of algorithms is important. Currently we recommend using the Histogram algorithm when analyzing code changes.

Open Access This article is distributed under the terms of the Creative Commons Attribution 4.0 International License (http://creativecommons.org/licenses/by/4.0/), which permits unrestricted use, distribution, and reproduction in any medium, provided you give appropriate credit to the original author(s) and the source, provide a link to the Creative Commons license, and indicate if changes were made.

\section{References}

Barr ET, Brun Y, Devanbu P, Harman M, Sarro F (2014) The plastic surgery hypothesis. In: Proceedings of the 22nd ACM SIGSOFT international symposium on foundations of software engineering, FSE 2014. ACM, New York, pp 306-317

Budgen D, Turner M, Brereton P, Kitchenham B (2008) Using mapping studies in software engineering. http://citeseerx.ist.psu.edu/viewdoc/summary?doi=10.1.1.222.9091

da Costa DA, McIntosh S, Shang W, Kulesza U, Coelho R, Hassan AE (2017) A framework for evaluating the results of the szz approach for identifying bug-introducing changes. IEEE Trans Softw Eng 43(7):641657. https://doi.org/10.1109/TSE.2016.2616306

Dotzler G, Philippsen M (2016) Move-optimized source code tree differencing. In: 2016 31st IEEE/ACM international conference on automated software engineering (ASE), pp 660-671

Duala-Ekoko E, Robillard MP (2007) Tracking code clones in evolving software. In: Proceedings of the 29th international conference on software engineering, ICSE '07. IEEE Computer Society, Washington, pp 158-167. https://doi.org/10.1109/ICSE.2007.90

Falleri JR, Morandat F, Blanc X, Martinez M, Monperrus M (2014) Fine-grained and accurate source code differencing. In: Proceedings of the 29th ACM/IEEE international conference on automated software engineering, ASE '14. ACM, New York, pp 313-324. https://doi.org/10.1145/2642937.2642982. http:// doi.acm.org/10.1145/2642937.2642982

Fluri B, Wuersch M, PInzger M, Gall H (2007) Change distilling: tree differencing for fine-grained source code change extraction. IEEE Trans Softw Eng 33(11):725-743. https://doi.org/10.1109/TSE.2007. 70731

Gousios G, Kalliamvakou E, Spinellis D (2008) Measuring developer contribution from software repository data. In: Proceedings of the 2008 international working conference on mining software repositories, MSR '08. ACM, New York, pp 129-132. https://doi.org/10.1145/1370750.1370781. http://doi.acm.org/ $10.1145 / 1370750.1370781$

Hashimoto M, Mori A (2008) Diff/ts: a tool for fine-grained structural change analysis. In: 2008 15th working conference on reverse engineering, pp 279-288. https://doi.org/10.1109/WCRE.2008.44

Hata H, Mizuno O, Kikuno T (2012) Bug prediction based on fine-grained module histories. In: Proceedings of the 34th international conference on software engineering, ICSE '12. IEEE Press, Piscataway, pp 200210. http://dl.acm.org/citation.cfm?id=2337223.2337247

Higo Y, Ohtani A, Kusumoto S (2017) Generating simpler ast edit scripts by considering copy-and-paste. In: Proceedings of the $32 \mathrm{Nd}$ IEEE/ACM international conference on automated on software engineering, ASE 2017. IEEE Press, Piscataway, pp 532-542

Huang K, Chen B, Peng X, Zhou D, Wang Y, Liu Y, Zhao W (2018) Cldiff: generating concise linked code differences. In: Proceedings of the 33rd ACM/IEEE international conference on automated software engineering, ASE 2018. ACM, New York, pp 679-690. https://doi.org/10.1145/3238147.3238219. http://doi.acm.org/10.1145/3238147.3238219

Hunt J, MacIlroy M (1976) An algorithm for differential file comparison. Computing science technical report, Bell Laboratories, https://books.google.com/books?id=zJ2LMwAACAAJ

Kamei Y, Shihab E (2016) Defect prediction: accomplishments and future challenges. In: 2016 IEEE 23rd International conference on software analysis, evolution, and reengineering (SANER), vol 5, pp 33-45. https://doi.org/10.1109/SANER.2016.56

Kavitha R (2009) Collection development in digital libraries: trends and problems. http://citeseerx.ist.psu. edu/viewdoc/summary?doi=10.1.1.924.7945\&rank=1 
Kim M, Sazawal V, Notkin D, Murphy G (2005) An empirical study of code clone genealogies. In: Proceedings of the 10th European software engineering conference held jointly with 13th ACM SIGSOFT international symposium on foundations of software engineering, ESEC/FSE-13. ACM, New York, pp 187-196. https://doi.org/10.1145/1081706.1081737. http://doi.acm.org/10.1145/1081706.1081737

Kim M, Notkin D, Grossman D, Wilson G (2013) Identifying and summarizing systematic code changes via rule inference. IEEE Trans Softw Eng 39(1):45-62. https://doi.org/10.1109/TSE.2012.16

Kuhrmann M, Fernandez MD, Daneva M (2017) On the pragmatic design of literature studies in software engineering: an experience-based guideline. Empir Softw Eng 22(6):2852-2891. https://doi.org/10.1007/ s10664-016-9492-y

Madeyski L, Jureczko M (2015) Which process metrics can significantly improve defect prediction models? an empirical study. Softw Qual J 23(3):393-422. https://doi.org/10.1007/s11219-014-9241-7

Meng X, Miller BP, Williams WR, Bernat AR (2013) Mining software repositories for accurate authorship. In: 2013 IEEE international conference on software maintenance, pp 250-259. https://doi.org/10.1109/ ICSM.2013.36

Myers EW (1986) An o(nd) difference algorithm and its variations. Algorithmica 1:251-266

Nagappan N, Ball T (2005) Use of relative code churn measures to predict system defect density. In: Proceedings of the 27th international conference on software engineering, ICSE '05. ACM, New York, pp 284-292. https://doi.org/10.1145/1062455.1062514. http://doi.acm.org/10.1145/1062455.1062514

Petersen K, Feldt R, Mujtaba S, Mattsson M (2008) Systematic mapping studies in software engineering. In: Proceedings of the 12th international conference on evaluation and assessment in software engineering, BCS Learning \& Development Ltd., Swindon, UK, EASE'08, pp 68-77. http://dl.acm.org/citation.cfm? id $=2227115.2227123$

Petersen K, Vakkalanka S, Kuzniarz L (2015) Guidelines for conducting systematic mapping studies in software engineering: an update. Inf Softw Technol 64:1-18. https://doi.org/10.1016/j.infsof.2015.03.007. http://www.sciencedirect.com/science/article/pii/S0950584915000646

Rahman F, Devanbu P (2011) Ownership, experience and defects: a fine-grained study of authorship. In: Proceedings of the 33rd international conference on software engineering, ICSE '11. ACM, New York, pp 491-500. https://doi.org/10.1145/1985793.1985860. http://doi.acm.org/10.1145/1985793.1985860

Rausch T, Hummer W, Leitner P, Schulte S (2017) An empirical analysis of build failures in the continuous integration workflows of java-based open-source software. In: Proceedings of the 14th international conference on mining software repositories, MSR '17. IEEE Press, Piscataway, pp 345-355, https://doi.org/10.1109/MSR.2017.54

Ray B, Nagappan M, Bird C, Nagappan N, Zimmermann T (2015) The uniqueness of changes: characteristics and applications. In: Proceedings of the 12th working conference on mining software repositories, MSR'15. IEEE Press, Piscataway, pp 34-44. http://dl.acm.org/citation.cfm?id=2820518.2820526

Rodriguez-Perez G, Robles G, Gonzalez-Barahona JM (2018) Reproducibility and credibility in empirical software engineering: a case study based on a systematic literature review of the use of the szz algorithm. Inf Softw Technol 99:164-176

Shin Y, Meneely A, Williams L, Osborne JA (2011) Evaluating complexity, code churn, and developer activity metrics as indicators of software vulnerabilities. IEEE Trans Softw Eng 37(6):772-787. https://doi.org/10.1109/TSE.2010.81

Śliwerski J, Zimmermann T, Zeller A (2005) When do changes induce fixes SIGSOFT Software Engineering Notes 30(4):1—5. https://doi.org/10.1145/1082983.1083147. http://doi.acm.org/10.1145/1082983. 1083147

Viera A, Garrett J (2005) Understanding interobserver agreement: the kappa statistic. Fam Med 37(5):360_ 363

Wohlin C, Runeson P, da Mota Silveira Neto PA, Engstrom E, do Carmo Machado I, de Almeida ES (2013) On the reliability of mapping studies in software engineering. Journal of Systems and Software 86(10):2594-2610. https://doi.org/10.1016/j.jss.2013.04.076. http://www.sciencedirect.com/ science/article/pii/S0164121213001234

Publisher's note Springer Nature remains neutral with regard to jurisdictional claims in published maps and institutional affiliations. 


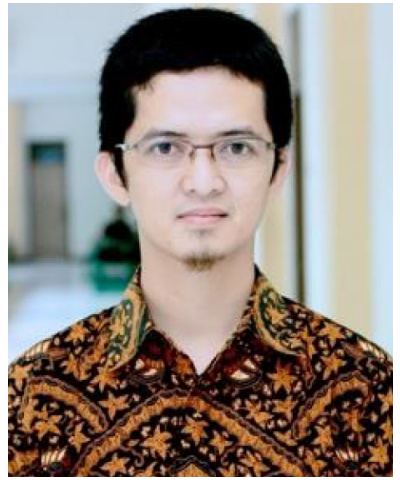

Yusuf Sulistyo Nugroho is a PhD student in the Graduate School of Science and Technology at the Nara Institute of Science and Technology. He received his M.Eng degree from Universitas Gadjah Mada, Indonesia. His research interests include mining software repositories and software ecosystems.

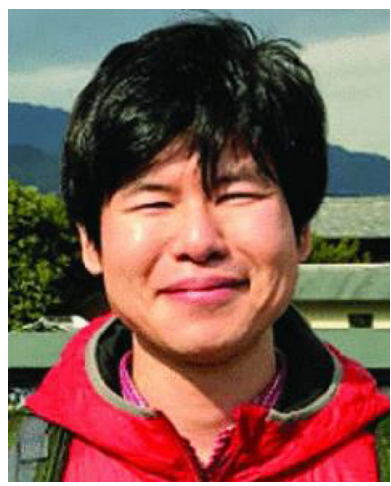

Hideaki Hata is an assistant professor at the Nara Institute of Science and Technology. His research interests include software ecosystems, human capital in software engineering, and software economics. He received a Ph.D. in information science from Osaka University. He is a Member of the IEEE and ACM. 


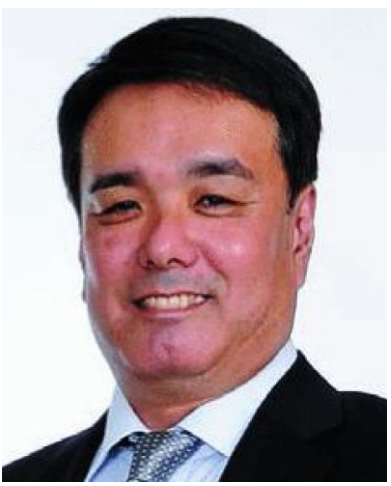

Kenichi Matsumoto is a professor in the Graduate School of Science and Technology at the Nara Institute of Science and Technology. His research interests include software measurement and software processes. He received a Ph.D. in information and computer sciences from Osaka University. He is a Senior Member of the IEEE and a member of the IEICE and the IPSJ. 